\title{
FLUX IDENTIFICATION FOR 1-d SCALAR CONSERVATION LAWS IN THE PRESENCE OF SHOCKS
}

\author{
CARLOS CASTRO AND ENRIQUE ZUAZUA
}

\begin{abstract}
We consider the problem of flux identification for 1-d scalar conservation laws formulating it as an optimal control problem. We introduce a new optimization strategy to compute numerical approximations of minimizing fluxes.

We first prove the existence of minimizers. We also prove the convergence of discrete minima obtained by means of monotone numerical approximation schemes, by a $\Gamma$-convergence argument. Then we address the problem of developing efficient descent algorithms. We first consider and compare the existing two possible approaches. The first one, the so-called discrete approach, based on a direct computation of gradients in the discrete problem and the so-called continuous one, where the discrete descent direction is obtained as a discrete copy of the continuous one. When optimal solutions have shock discontinuities, both approaches produce highly oscillating minimizing sequences and the effective descent rate is very weak. As a remedy we adapt the method of alternating descent directions that uses the recent developments of generalized tangent vectors and the linearization around discontinuous solutions, introduced by the authors, in collaboration with F. Palacios, in the case where the control is the initial datum. This method distinguishes descent directions that move the shock and those that perturb the profile of the solution away from it. As we shall see, a suitable alternating combination of these two classes of descent directions allows building more efficient and faster descent algorithms.
\end{abstract}

\section{INTRODUCTION}

The optimal control of hyperbolic conservation laws is a difficult topic both from the analytical and computational point of view. One of the main difficulties is that classical analysis usually fails due to the presence of discontinuous solutions. In the recent years a number of methods have been proposed to deal with these singular solutions, to reduce the computational cost and to render these types of problems affordable, both for scalar equations and systems (see [1, [2, 10], 11, [16], 19], 21], 26, 35]).

In this article, we focus on the simpler scalar conservation law:

$$
\left\{\begin{array}{l}
\partial_{t} u+\partial_{x}(f(u))=0, \quad \text { in } \mathbb{R} \times(0, T), \\
u(x, 0)=u^{0}(x), \quad x \in \mathbb{R}
\end{array}\right.
$$

$T>0$ being a given finite time horizon.

Received by the editor June 10, 2009 and, in revised form, July 12, 2010.

2010 Mathematics Subject Classification. Primary 49J20; Secondary 90C31, 65K10.

Key words and phrases. Flux identification, 1-d scalar conservation laws, optimal control, numberical approximation, alternating descent method.

This work was supported by the Grant MTM2008-03541 of the MICINN (Spain). 
We address the problem of flux identification in which the flux, which is the nonlinearity $f$ of the equation, is treated as a control parameter.

To fix ideas, given an initial datum $u^{0} \in B V(\mathbb{R}) \cap L^{\infty}(\mathbb{R}) \cap L^{1}(\mathbb{R})$ and a target $u^{d} \in L^{2}(\mathbb{R})$, we consider the cost functional to be minimized as $J: C^{1}(\mathbb{R}) \rightarrow \mathbb{R}$, defined by

$$
J(f)=\frac{1}{2} \int_{\mathbb{R}}\left|u(x, T)-u^{d}(x)\right|^{2} d x,
$$

where $u(x, t) \in L^{\infty}(\mathbb{R} \times(0, T)) \cap C\left([0, T] ; L^{1}(\mathbb{R})\right)$ is the unique entropy solution of (1.1).

This problem has been previously studied in [26] where particular applications to identification of mixture concentrations in chromatography are presented. We also refer to [25] where a complete description of the mathematical modeling of such problems and their numerical approximation is given.

Although this paper is devoted to the particular choice of $J$ in (1.2), most of our analysis and numerical algorithms can be adapted to many other functionals and control problems.

We also introduce the set of admissible nonlinearities $\mathcal{U}_{a d} \subset C^{1}(\mathbb{R})$, that we will make precise later in order to guarantee the existence of minimizers for the following optimization problem: Find $f^{\text {min }} \in \mathcal{U}_{a d}$ such that,

$$
J\left(f^{\min }\right)=\min _{f \in \mathcal{U}_{a d}} J(f) .
$$

As we will see, the existence of minimizers can be easily established under some natural assumptions on the class of admissible nonlinearities $\mathcal{U}_{a d}$ using well-known well-posedness and compactness properties of solutions of scalar conservation laws. However, uniqueness is false, in general, due, in particular, to the possible presence of discontinuities in the solutions of (1.1). We show that these discontinuities may also affect the performance of the optimization algorithms.

In practical applications and in order to perform numerical computations and simulations one has to replace the continuous optimization problem above by a discrete one. It is then natural to consider a discretization of system (1.1) and the functional $J$. If this is done in an appropriate way, the discrete optimization problem has minimizers that are often taken, for small enough mesh-sizes, as approximations of the continuous minimizers. There are, however, few results in the context of hyperbolic conservation laws proving rigorously the convergence of the discrete optimal controls towards the continuous ones, as the mesh-size goes to zero.

One of the first goals of this paper is to provide such a convergence result based on the fine use of the known properties of monotone conservative schemes.

In the following we will denote by $u_{\Delta}$ the approximation of $u$ obtained by a suitable discretization of system (1.1) with mesh-sizes $\Delta x$ and $\Delta t$ for space-time discretizations. We also denote by $J_{\Delta}$ a discretization of $J$ and by $\mathcal{U}_{a d, \Delta}$ a discrete version of the set of admissible fluxes, or controls, $\mathcal{U}_{a d}$, and consider the approximate discrete minimization problem,

$$
J_{\Delta}\left(f_{\Delta}^{\min }\right)=\min _{f_{\Delta} \in \mathcal{U}_{a d, \Delta}} J_{\Delta}\left(f_{\Delta}\right) .
$$

For fixed values of the mesh-size $\Delta$, the existence of minimizers for this discrete problem is often easy to prove. But, even in that case, their convergence as $\Delta \rightarrow 0$ is harder to show. This will be done, as mentioned above, in the class of monotone schemes. 
From a practical point of view it is also important to be able to develop efficient algorithms for computing accurate approximations of the discrete minimizers. This is often not an easy matter due to the large number of parameters involved, the lack of convexity of the functional under consideration, etc.

The most frequently used methods to approximate minimizers are the gradient ones (steepest descent, conjugate gradient, etc.), although they hardly distinguish local and global minimizers. This is an added difficulty in problems with many local minima, a fact that cannot be excluded in our optimization problem, due to the nonlinear dependence of the state on the initial datum. However, we will not address this problem here. We shall instead focus on building efficient descent algorithms.

As we have said, this flux identification problem has been previously studied in [26. Also, some numerical methods are discussed to solve the optimization problem but they are based on a classical approach that can be justified only for smooth solutions. The main contribution of this paper is that we deal with solutions having discontinuities. In fact, we propose a new optimization technique where the position of the discontinuity is treated as a new variable in the optimization process.

The convergence of an algorithm based on this idea is a difficult and interesting open problem. To understand it in more detail we observe that our strategy can be interpreted as a relaxation method in finite dimensions where partial derivatives are used to descend in a cyclic way. In our case, we propose a decomposition of the gradient in two vectors that are used alternatively. One of these components concerns the position of the discontinuity and the other the usual smooth variation to both sides of the discontinuity. Convergence of relaxation methods in finite dimensions is known under convexity and regularity assumptions on the cost functional (see [18]). A generalization to the present situation could be obtained with similar hypotheses on the cost functional. However, the cost functional under consideration is neither convex nor differentiable.

The rest of the paper is organized as follows: In section 2 we prove the existence of minimizers for problem (1.3). In section 3 we prove the convergence of the discrete minimizers of (1.4) to minimizers of the continuous problem. In section 4 we analyze the sensitivity of the continuous functional (1.2). We analyze it both for smooth solutions and for solutions having a single isolated shock. In section 5 we introduce a new characterization of the sensitivity of the functional which allows us to identify variations that do not move the shock position. This allows us to define a new optimization strategy which is the main contribution of this paper. In section 6 we show how to implement numerically a gradient algorithm to solve the discrete optimization problem. In particular, we use the sensitivities computed in the previous sections. Section 7 is devoted to show some numerical experiments which illustrate the efficiency of the different optimization methods described in the paper. Section 8 contains some concluding remarks. In the Appendix at the end of the paper we prove the linearization result for the scalar conservation law, with respect to the nonlinearity, in the presence of shocks.

\section{EXISTENCE OF MINIMIZERS}

In this section we state that, under certain conditions on the set of admissible nonlinearities $\mathcal{U}_{a d}$, there exists at least one minimizer of the functional $J$ given in (1.2). 
We assume $u^{0} \in B V(\mathbb{R}) \cap L^{\infty}(\mathbb{R}) \cap L^{1}(\mathbb{R})$. The Kruzkov theorem states the existence and uniqueness of a unique entropy solution $u(x, t)$ of (1.1) in the class $u \in B V(\mathbb{R} \times(0, T)) \cap C^{0}\left([0, T] ; L_{\text {loc }}^{1}(\mathbb{R})\right)$ (see [27], 7]). Note that, in our case, the solution also belongs to $C^{0}\left([0, T] ; L^{1}(\mathbb{R})\right)$. From the maximum principle (see for instance [19], p. 60), this unique entropy solution $u(x, t)$ satisfies the upper bound

$$
\|u(\cdot, t)\|_{L^{\infty}(\mathbb{R})} \leq\left\|u^{0}\right\|_{L^{\infty}(\mathbb{R})} .
$$

Thus, only the values of the nonlinearity $f$ in the interval $\left[-\left\|u^{0}\right\|_{L^{\infty}},\left\|u^{0}\right\|_{L^{\infty}}\right]$ are relevant to solve (1.1). According to this, the restrictions we will impose in the fluxes, if any, only make sense within that bounded range.

We consider the class of admissible fluxes $\mathcal{U}_{a d}$ : $(2.2) \mathcal{U}_{a d}=\left\{f \in W^{2, \infty}\left(\left[-\left\|u^{0}\right\|_{L^{\infty}},\left\|u^{0}\right\|_{L^{\infty}}\right]\right):\|f\|_{W^{2, \infty}\left(\left[-\left\|u^{0}\right\|_{L^{\infty}},\left\|u^{0}\right\|_{L^{\infty}}\right]\right)} \leq C\right\}$, where $C>0$ is a constant. Note that $\mathcal{U}_{a d}$ is a compact set with respect to the topology of $W^{1, \infty}\left(\left[-\left\|u^{0}\right\|_{L^{\infty}},\left\|u^{0}\right\|_{L^{\infty}}\right]\right)$, since the interval $\left[-\left\|u^{0}\right\|_{L^{\infty}},\left\|u^{0}\right\|_{L^{\infty}}\right]$ is bounded.

On the other hand, in [29] it is proved that the application $f \rightarrow u_{f}$, where $u_{f}$ is the unique entropy solution of (1.1) with initial datum $u^{0}$, satisfies

$$
\left\|u_{f}(\cdot, t)-u_{g}(\cdot, t)\right\|_{L^{1}(\mathbb{R})} \leq t\|f-g\|_{L i p}\left\|u^{0}\right\|_{B V(\mathbb{R})} .
$$

This continuity result for the solutions of (1.1) with respect to the fluxes $f$, provides easily the following existence result (see [26] for details):

Theorem 2.1. Assume that $u^{0} \in B V(\mathbb{R}) \cap L^{\infty}(\mathbb{R}) \cap L^{1}(\mathbb{R}), u^{d} \in L^{2}(\mathbb{R})$ and $\mathcal{U}_{a d}$ is as in (2.2). Then the minimization problem,

$$
\min _{f \in \mathcal{U}_{a d}} J(f),
$$

has at least one minimizer $f^{\text {min }} \in \mathcal{U}_{\text {ad }}$.

Uniqueness is, in general, false for this optimization problem.

Proof. The existence of a minimizer is easily obtained from the direct method of the calculus of variations. We briefly sketch the proof.

Let us consider a minimizing sequence $\left\{f_{j}\right\}_{j>1}$ in $\mathcal{U}_{a d}$. Since $\mathcal{U}_{a d}$ is bounded in $W^{2, \infty}\left(\left[-\left\|u^{0}\right\|_{L^{\infty}},\left\|u^{0}\right\|_{L^{\infty}}\right]\right)$, the sequence $f_{j}$ contains a subsequence, still denoted $f_{j}$, such that $f_{j} \rightarrow f$ weakly- ${ }^{*}$ in $W^{2, \infty}\left(\left[-\left\|u^{0}\right\|_{L^{\infty}},\left\|u^{0}\right\|_{L^{\infty}}\right]\right)$ and strongly in $W^{1, \infty}\left(\left[-\left\|u^{0}\right\|_{L^{\infty}},\left\|u^{0}\right\|_{L^{\infty}}\right]\right)$. Obviously, $f \in \mathcal{U}_{a d}$ and it is a minimizer if we show that $\lim _{j \rightarrow \infty} J\left(f_{j}\right)=J(f)$.

Let $u_{j}$ be the unique entropy solution of (1.1) with numerical flux $f_{j}$. Then, as we mentioned above, only the values of $f_{j}$ on the bounded set $\left[-\left\|u^{0}\right\|_{L^{\infty}},\left\|u^{0}\right\|_{L^{\infty}}\right]$ are relevant to obtain $u_{j}$, and therefore to evaluate $J$. This, together with the continuity result in (2.3), provides the continuity of $J$.

The lack of uniqueness of minimizers can be easily checked for some specific choices of the initial datum. This can be easily seen observing that the flux $f$ only affects the slope of the characteristics associated to (1.1) on the range of $u$. Thus, for example, the shock wave function

$$
u(x, t)=\left\{\begin{array}{l}
1 \text { if } x \leq t / 2, \\
0 \text { if } x>t / 2,
\end{array}\right.
$$

which only takes two values $u=0,1$, solves (1.1) whatever $f$ is, provided $f^{\prime}(0)=0$, $f^{\prime}(1)=1$ and $f(1)-f(0)=1 / 2$. If we take $u^{0}=u(x, 0)$ and $u^{d}=u(x, T)$, all of these fluxes $f$ are minimizers of (2.4) for which $J(f)=0$. 
Remark 2.1. The proof above can be slightly modified to deal with larger classes of admissible nonlinearities $\mathcal{U}_{a d}$. For instance, $\mathcal{U}_{a d} \subset W^{1, \infty}\left(\left[-\left\|u^{0}\right\|_{L^{\infty}},\left\|u^{0}\right\|_{L^{\infty}}\right]\right)$ with the bound $\left\|f^{\prime}\right\|_{L^{\infty}\left(\left[-\left\|u^{0}\right\|_{L \infty},\left\|u^{0}\right\|_{L \infty}\right]\right)} \leq C$. The main difficulty in this case is that we cannot apply the Lipschitz dependence property (2.3) to deduce the $L^{1}$ convergence of solutions, when considering a minimizing sequence $f_{j} \rightarrow f$. Instead, we use the uniform convergence $f_{j} \rightarrow f$ which holds, at least for a subsequence. Then, it is possible to pass to the limit in the weak formulation

$$
\int_{0}^{T} \int_{\mathbb{R}}\left(u_{j} \psi_{t}+f_{j}\left(u_{j}\right) \psi_{x}\right) d x d t+\int_{\mathbb{R}} u^{0}(x) \varphi(x, 0)=0, \quad \forall \psi \in C_{c}^{1}(\mathbb{R} \times[0, T)),
$$

since the solutions $u_{j}$ converge a.e. The latter is guaranteed by the uniform $B V$ estimate on the solutions consequence of the $L^{1}$-contraction property and the fact that the initial data are taken to be in $B V$. Obviously, since we are dealing with the unique entropy solutions, a similar argument is needed to pass to the limit on the weak form of the entropy condition. In this way, we obtain the convergence $u_{j} \rightarrow u$ in $L_{l o c}^{1}(\mathbb{R} \times(0, T))$. The convergence of $u_{j}(\cdot, T) \rightarrow u(\cdot, T)$ in $L_{l o c}^{1}(\mathbb{R})$ follows from the uniform $B V$ estimate of $u_{j}(\cdot, T)$. Finally, the convergence in $L^{1}(\mathbb{R})$ can be deduced by proving that the integral of $\left|u_{j}(\cdot, T)\right|$ on $(-\infty,-K) \cup(K, \infty)$ is small for large $K$, uniformly in $j$. This is easily obtained arguing with the domain of dependence of $u_{j}(x, T)$ in $x \in(-\infty,-K) \cup(K, \infty)$, the $L^{1}$-contraction and the fact that $u_{j}(x, 0)=u^{0}(x) \in L^{1}(\mathbb{R})$.

In practice, we can also remove the $W^{1, \infty}$ bound in the admissible set $\mathcal{U}_{a d}$ by including a regularization term in the functional $J$, i.e., we can consider $\mathcal{U}_{a d}=$ $W^{1, \infty}\left(\left[-\left\|u^{0}\right\|_{L^{\infty}},\left\|u^{0}\right\|_{L^{\infty}}\right]\right)$ and the penalized functional

$$
J(f)=\frac{1}{2} \int_{\mathbb{R}}\left|u(x, T)-u^{d}(x)\right|^{2} d x+K \int_{0}^{1}\left|f^{\prime}(s)\right| d s
$$

for some penalization parameter $K>0$. The same arguments as before allow proving the existence of minimizers for this functional but, this time, without imposing a priori bounds on the fluxes $f$.

In practical applications, the above infinite dimensional optimization problem is usually reduced to a finite dimensional one by considering a finite dimensional subspace of the class of admissible fluxes $\mathcal{U}_{a d}$, that we denote by $\mathcal{U}_{a d}^{M}$. More precisely, we take $\mathcal{U}_{a d}^{M}$ as a subset of the set of linear combinations of some, a priori chosen, smooth basis functions $f_{1}, \ldots, f_{M} \in W^{2, \infty}\left(\left[-\left\|u^{0}\right\|_{L^{\infty}},\left\|u^{0}\right\|_{L^{\infty}}\right]\right)$,

$$
\begin{aligned}
\mathcal{U}_{a d}^{M}= & \left\{f=\sum_{j=1}^{M} \alpha_{j} f_{j}, \quad \text { with } \alpha_{j} \in \mathbb{R} \text { for all } j=1, \ldots, M,\right. \\
& \text { and } \left.\|f\|_{W^{2, \infty}\left(\left[-\left\|u^{0}\right\|_{L^{\infty}},\left\|u^{0}\right\|_{\left.L^{\infty}\right]}\right]\right.} \leq C\right\} .
\end{aligned}
$$

The reduced minimization problem can be stated as follows: Find $f^{\text {min }} \in \mathcal{U}_{a d}^{M}$ such that

$$
J\left(f^{\min }\right)=\min _{f \in \mathcal{U}_{a d}^{M}} J(f) .
$$

Of course, the arguments in the proof of Theorem 2.1 allow showing that the minimizer of (2.7) exists as well for all finite $M$. But this time things can be done more easily since the space of possible designs/controls is finite-dimensional. More precisely, the bound $\|f\|_{W^{2, \infty}\left(\left[-\left\|u^{0}\right\|_{L \infty},\left\|u^{0}\right\|_{L} \infty\right]\right)} \leq C$ in the set of admissible controls can be relaxed to any other one guaranteeing the boundedness of the scalar 
coefficients $\alpha_{j}, i=1, \ldots, M$, in the linear combination of the reference fluxes under consideration.

This is in fact the problem that we approximate numerically in the following section.

\section{THE DISCRETE MINIMIZATION PROBLEM}

The purpose of this section is to show that discrete minimizers obtained through suitable numerical schemes, converge to a minimizer of the continuous problem (2.7), as the mesh-size tends to zero. This justifies the usual engineering practice of replacing the continuous functional and model by discrete ones to compute an approximation of the continuous minimizer.

We introduce a suitable discretization of the functional $J$ and the conservation law (1.1). Let us consider a mesh in $\mathbb{R} \times[0, T]$ given by $\left(x_{j}, t^{n}\right)=(j \Delta x, n \Delta t)$ $(j=-\infty, \ldots, \infty ; n=0, \ldots, N+1$ so that $(N+1) \Delta t=T)$, and let $u_{j}^{n}$ be a numerical approximation of $u\left(x_{j}, t^{n}\right)$ obtained as the solution of a suitable discretization of the equation (1.1).

Let $u_{\Delta}^{0}=\left\{u_{j}^{0}\right\}$ be the discrete initial datum, which is taken to be an approximation of the initial datum $u^{0}$ of the continuous conservation law. Similarly, let $u_{\Delta}^{d}=\left\{u_{j}^{d}\right\}$ be the discretization of the target $u^{d}$ at $x_{j}$. A common choice is to take,

$$
u_{j}^{0}=\frac{1}{\Delta x} \int_{x_{j-1 / 2}}^{x_{j+1 / 2}} u^{0}(x) d x, \quad u_{j}^{d}=\frac{1}{\Delta x} \int_{x_{j-1 / 2}}^{x_{j+1 / 2}} u^{d}(x) d x,
$$

where $x_{j \pm 1 / 2}=x_{j} \pm \Delta x / 2$.

We also consider the following approximation of the functional $J$ in (1.2):

$$
J_{\Delta}(f)=\frac{\Delta x}{2} \sum_{j=-\infty}^{\infty}\left(u_{j}^{N+1}-u_{j}^{d}\right)^{2}
$$

In order to compute $u_{j}^{n}$ from $f$ and $u_{\Delta}^{0}$, we introduce a 3-point conservative numerical approximation scheme for (1.1):

$$
u_{j}^{n+1}=u_{j}^{n}-\lambda\left(g_{j+1 / 2}^{n}-g_{j-1 / 2}^{n}\right), \quad \lambda=\frac{\Delta t}{\Delta x}, \quad j \in \mathbb{Z}, n=0, \ldots, N,
$$

where,

$$
g_{j+1 / 2}^{n}=g\left(u_{j}^{n}, u_{j+1}^{n}\right),
$$

and $g$ is a Lipschitz continuous function usually referred to as the numerical flux. These schemes are consistent with (1.1) when $g(u, u)=f(u)$.

As usual, in order to define convergence results of discrete solutions, we introduce the piecewise constant function $u_{\Delta}$ defined in $\mathbb{R} \times[0, T]$ by

$$
u_{\Delta}(x, t)=u_{j}^{n}, \quad x \in\left(x_{j-1 / 2}, x_{j+1 / 2}\right), \quad t \in\left[t^{n}, t^{n+1}\right) .
$$

We assume that the numerical schemes also satisfy the following two properties:

(1) The following discrete version of the maximum principle in (2.1):

$$
\left\|u_{\Delta}(x, t)\right\|_{L^{\infty}} \leq\left\|u_{\Delta}^{0}\right\|_{L^{\infty}}, \quad \forall n \geq 0 .
$$

(2) The discrete solutions converge to weak entropy solutions of the continuous conservation law, as the discretization parameter $\Delta x$ tends to zero, with some fixed $\lambda=\Delta t / \Delta x$, under a suitable CFL condition. 
The so-called monotone schemes (see [19, Chap. 3) constitute a particular class of conservative numerical methods that satisfy these conditions. Recall that a method is said to be monotone if the function $H(u, v, w)=v-\lambda(g(v, w)-g(u, v))$ is a monotone increasing function in each argument. Some examples of monotone methods are the Lax-Friedrichs, Engquist-Osher and Godunov schemes, under a suitable CFL condition which depends on each method. Their numerical fluxes are given, respectively, by

$$
\begin{aligned}
g^{L F}(u, v) & =\frac{f(u)+f(v)}{2}-\frac{v-u}{2 \lambda}, \\
g^{E O}(u, v) & =\frac{1}{2}\left(f(u)+f(v)-\int_{u}^{v}\left|f^{\prime}(s)\right| d s\right), \\
g^{G o d}(u, v) & = \begin{cases}\min _{w \in[u, v]} f(w), & \text { if } u \leq v, \\
\max _{w \in[v, u]} f(w), & \text { if } u \geq v .\end{cases}
\end{aligned}
$$

For example, the CFL condition for the Lax-Friedrichs scheme is as follows (see [20], p. 139),

$$
\lambda \max _{j}\left|\frac{f\left(u_{j+1}\right)-f\left(u_{j}\right)}{u_{j+1}-u_{j}}\right| \leq 1 .
$$

In the sequel we make explicit the dependence of the numerical flux $g$ on $f$ by writing $g(u, v ; f)$. We also assume that the numerical flux $g(u, v ; f)$ depends continuously on $f \in \mathcal{U}_{a d}^{M}$, with respect to the $W^{2, \infty}$ norm, and that the Lipschitz constant of $g$ is independent of $f$, i.e.,

$$
\frac{\left|g\left(u_{1}, v_{1} ; f\right)-g\left(u_{2}, v_{2} ; f\right)\right|}{\left|u_{1}-u_{2}\right|+\left|v_{1}-v_{2}\right|} \leq C, \quad \forall u_{i}, v_{i} \in\left[-\left\|u^{0}\right\|_{L^{\infty}},\left\|u^{0}\right\|_{L^{\infty}}\right],
$$

with a constant $C$ independent of $f \in \mathcal{U}_{a d}^{M}$. Note that in (3.8) it is enough to consider values of $u, v$ in a finite interval. In fact, the discrete maximum principle (3.4) ensures the boundedness of the discrete solutions regardless of the chosen flux $f \in \mathcal{U}_{a d}^{M}$.

We then consider the following discrete minimization problem: Find $f_{\Delta}^{\min } \in \mathcal{U}_{a d}^{M}$ such that

$$
J_{\Delta}\left(f_{\Delta}^{\min }\right)=\min _{f \in \mathcal{U}_{a d}^{M}} J_{\Delta}(f),
$$

where $\mathcal{U}_{a d}^{M}$ is as in (2.6).

For each $\Delta x, \Delta t>0$ it is easy to see that the discrete analogue of the existence result in Theorem 2.1 holds. Moreover, the uniform bound (3.8) and formula (2.3) allow also to pass to the limit as $\Delta x, \Delta t \rightarrow 0$.

Theorem 3.1. Assume that the hypotheses of Theorem 2.1 are satisfied. Assume also that $u_{\Delta}$ is obtained by a conservative monotone numerical scheme consistent with (1.1) and that the associated numerical flux $g(u, v ; f)$ satisfies (3.8) and it depends continuously on $f \in \mathcal{U}_{a d}^{M}$. Then:

- For all $\Delta x, \Delta t>0$, the discrete minimization problem (3.9) has at least one solution $f_{\Delta}^{\min } \in \mathcal{U}_{a d}^{M}$.

- Any accumulation point of $f_{\Delta}^{\min } \in \mathcal{U}_{a d}^{M}$ as $\Delta x, \Delta t \rightarrow 0$ (with $\Delta t / \Delta x=\lambda$ fixed and under the corresponding CFL condition), is a minimizer of the continuous problem (2.4) in $\mathcal{U}_{a d}^{M}$. 
Proof. For fixed $\Delta x$ and $\Delta t$ the proof of the existence of minimizers can be done as in the continuous case.

We now address the limit problem $\Delta x \rightarrow 0$, when $\lambda=\Delta t / \Delta x$ is fixed that, for simplicity, we denote by $\Delta \rightarrow 0$.

We follow a standard $\Gamma$-convergence argument. The key ingredient is the following continuity property: Assume that $\left\{f_{j}\right\}_{j \geq 1} \subset \mathcal{U}_{a d}^{M}$ satisfies $f_{j} \rightarrow f$ weakly-* in $W^{2, \infty}\left(\left[-\left\|u^{0}\right\|_{L^{\infty}},\left\|u^{0}\right\|_{L^{\infty}}\right]\right)$ and strongly in $W^{1, \infty}\left(\left[-\left\|u^{0}\right\|_{L^{\infty}},\left\|u^{0}\right\|_{L^{\infty}}\right]\right)$. Then

$$
\lim _{(j, \Delta) \rightarrow(\infty, 0)} J_{\Delta}\left(f_{j}\right)=J(f) .
$$

In the particular case under consideration, the convergence assumption on the nonlinearities is equivalent to the convergence of the finite dimensional parameters $\alpha_{j}$ entering in the definition of the set of admissible controls $\mathcal{U}_{a d}^{M}$.

The continuity property (3.10) is easily obtained from the estimate,

$$
\left|J_{\Delta}\left(f_{j}\right)-J(f)\right| \leq\left|J_{\Delta}\left(f_{j}\right)-J\left(f_{j}\right)\right|+\left|J\left(f_{j}\right)-J(f)\right| .
$$

The second term on the right-hand side converges to zero, as $j \rightarrow \infty$, due to the continuity of $J$ stated in the proof of Theorem 2.1. Concerning the first term, we use the convergence of the discrete solutions obtained with monotone numerical schemes, as $\Delta \rightarrow 0$, under the CFL condition, in the $L^{\infty}\left(0, T ; L^{2}(\mathbb{R})\right)$ norm (see, for example, [19]). In fact, following the argument in [19] (Theorem 3.4, p. 132), it is easy to check that this convergence is uniform with respect to the fluxes $f \in \mathcal{U}_{a d}^{M}$ under the uniform bound (2.6).

Now, let $\hat{f} \in \mathcal{U}_{a d}^{M}$ be an accumulation point of the minimizers of $J_{\Delta}, f_{\Delta}^{\min }$, as $\Delta \rightarrow 0$. To simplify the notation we still denote by $f_{\Delta}^{\min }$ the subsequence for which $f_{\Delta}^{\min } \rightarrow \hat{f}$, as $\Delta x, \Delta t \rightarrow 0$. Let $h \in \mathcal{U}_{a d}^{M}$ be any other function. We are going to prove that

$$
J(\hat{f}) \leq J(h)
$$

Taking into account the continuity property (3.10), we have

$$
J(h)=\lim _{\Delta \rightarrow 0} J_{\Delta}(h) \geq \lim _{\Delta \rightarrow 0} J_{\Delta}\left(f_{\Delta}^{\min }\right)=J(\hat{f}),
$$

which proves (3.11).

Remark 3.1. Using the fact that the initial datum $u^{0}$ is assumed to be in $B V$, the same existence and convergence results may be proved within larger classes of admissible sets $\mathcal{U}_{a d}$ and not only in the finite dimensional one $\mathcal{U}_{a d}^{M}$. For the existence of minimizers we can mimic the argument in Remark 2.1 as soon as $\mathcal{U}_{a d} \subset W^{1, \infty}\left(-\left\|u^{0}\right\|_{L^{\infty}},\left\|u^{0}\right\|_{L^{\infty}}\right)$ guarantees the minimum compactness properties so that the nonlinearities converge uniformly on bounded sets.

Concerning the convergence of minimizers, the main difficulty is to establish the uniform convergence of discrete solutions $u_{\Delta}$ in $L^{\infty}\left(0, T ; L^{2}(\mathbb{R})\right)$ with respect to the fluxes in $\mathcal{U}_{a d}$. But, as we said in the proof above, this is a consequence of the uniform bound (2.6). In fact, the $B V$ assumption on the initial datum and the monotonicity of the numerical schemes under consideration, ensure uniform $B V$ bounds on the solutions, both discrete and continuous ones, and this for all nonlinearities. This allows proving the uniform convergence of $u_{\Delta} \rightarrow u$ in $L_{l o c}^{1}(\mathbb{R})$ for any $t \in[0, T]$. Then, arguing with the domain of dependence of discrete solutions and the fact that $u^{0} \in L^{1}(\mathbb{R})$ it is easy to obtain the uniform convergence $u_{\Delta}(\cdot, t) \rightarrow u(\cdot, t)$ in $L^{1}(\mathbb{R})$. Finally, the uniform bound in $L^{\infty}$ provides the convergence in $L^{2}(\mathbb{R})$. 
Remark 3.2. Theorem 3.1 concerns global minima. However, both the continuous and discrete functionals may possibly have local minima as well. Extending this kind of $\Gamma$-convergence result for local minima requires further important developments.

\section{Sensitivity AnAlysis: The CONTINUOUs APPROACH}

In this section we describe and analyze the continuous approach to obtain descent directions for the discrete functional $J_{\Delta}$. We recall that in this case we use a suitable discretization of the steepest descent direction obtained for the continuous functional $J$ on the subset $\mathcal{U}_{a d}^{M}$.

The set $\mathcal{U}_{a d}^{M}$ can be parametrized by the vector of components $\alpha=\left(\alpha_{1}, \ldots, \alpha_{M}\right) \in$ $\mathbb{R}^{M}$ with values on the subset determined by the $W^{2, \infty}$ bound of the elements in $\mathcal{U}_{a d}^{M}$. Thus, a natural way to impose this constraint is to assume that $\mathcal{A}^{M}$ is given by,

$$
\mathcal{A}^{M}=\left\{\left(\alpha_{1}, \ldots, \alpha_{M}\right) \in \mathbb{R}^{M} \text { such that }\left|\alpha_{i}\right| \leq \gamma_{i}, i=1, \ldots, M\right\},
$$

for some constants $\gamma_{i}>0$. The optimization problem (2.7) is then reduced to the constrained minimization problem: Find $\alpha_{\min }$ such that,

$$
J\left(\alpha_{\min }\right)=\min _{\alpha \in \mathcal{A}^{M}} J(\alpha) .
$$

This optimization problem is usually written as an unconstrained problem for an augmented cost function $\tilde{J}(\alpha)=J(\alpha)+\sum_{i=1}^{M} \lambda_{i} \alpha_{i}$ which incorporates the Lagrange multiplier $\lambda=\left(\lambda_{1}, \ldots, \lambda_{M}\right) \in \mathbb{R}^{M}$. Note, however, that the main difficulty to obtain a descent direction for the augmented functional comes from the computation of the gradient of $J$, an issue that we address now.

We divide this section into three more subsections: More precisely, in the first one we consider the case where the solution $u$ of (1.1) has no shocks, in the second and third subsections we analyze the sensitivity of the solution and the functional in the presence of a single shock located on a regular curve.

4.1. Sensitivity without shocks. In this subsection we give an expression for the sensitivity of the functional $J$ with respect to the flux based on a classical adjoint calculus for smooth solutions.

Let $f \in C^{2}(\mathbb{R})$. Let $C_{0}^{1}(\mathbb{R})$ be the set of $C^{1}$ functions with compact support and let $u^{0} \in C_{0}^{1}(\mathbb{R})$ be a given datum for which there exists a classical solution $u(x, t)$ of (1.1) in $(x, t) \in \mathbb{R} \times[0, T+\tau]$, for some $\tau>0$. Note that this imposes some restrictions on $u^{0}$ and $f$ other than being smooth. More precisely, we must have $T+\tau<-1 / \min \left(\inf _{x}\left(f^{\prime \prime}\left(u_{0}(x)\right) u_{0}^{\prime}(x)\right), 0\right)$ to guarantee that two different characteristics do not meet in the time interval $[0, T+\tau]$.

The following holds:

Theorem 4.1. Under the above assumptions on $u^{0}, f$ and $T$, given any function $\delta f \in C^{2}(\mathbb{R})$, the Gateaux derivative of $J$ in the direction of $\delta f$ is given by

$$
\delta J(f)(\delta f)=-T \int_{\mathbb{R}} \partial_{x}(\delta f(u(x, T)))\left(u(x, T)-u^{d}(x)\right) d x .
$$

Remark 4.1. Formula (4.3) establishes, in particular, that only the values of $\delta f$ in the range of $u$ at time $t=T$ are relevant for the Gateaux derivative of the functional $J$. 
Remark 4.2. Note that for classical solutions the Gateaux derivative of $J$ at $f$ is given by (4.3) and this provides a descent direction for $J$ at $f$, as we have already seen. However, this is not very useful in practice since, even when we initialize the iterative descent algorithm with $f$ so that the corresponding solution $u$ is smooth, we cannot guarantee that the solution will remain classical along the iteration.

Corollary 4.1. Assume that the hypotheses of Theorem 4.1 hold. Assume also that the fluxes $f$ are chosen in the finite dimensional subspace parametrized by $\mathcal{A}^{M}$ in (4.1). The derivative of the functional $J$ at $\alpha$ in the tangent direction $\delta \alpha=\left(\delta \alpha_{1}, \ldots, \delta \alpha_{M}\right) \in \mathbb{R}^{M}$ is given by

$$
\delta J(\alpha)(\delta \alpha)=-T \sum_{m=1}^{M} \delta \alpha_{m} \int_{\mathbb{R}} \partial_{x}\left(f_{m}(u(x, T))\right)\left(u(x, T)-u^{d}(x)\right) d x .
$$

Thus, in the absence of constraints, the steepest descent direction for $J$ at $\alpha$ is given by

$$
\delta \alpha_{m}=\int_{\mathbb{R}} \partial_{x}\left(f_{m}(u(x, T))\right)\left(u(x, T)-u^{d}(x)\right) d x, \quad \text { for all } m \in 1, \ldots, M .
$$

Remark 4.3. Formula (4.3) allows us to also deduce a descent direction for $J(f)$ when we deal with the infinite dimensional space $\mathcal{U}_{a d}$, at least in some particular cases. For example, assume that the solution $u$ of (1.1) satisfies the following condition: there exists a finite set of intervals $\left\{I_{i}\right\}_{i=1}^{I}$ where $u(\cdot, T)$ is a strictly monotone function, i.e.,

$$
\partial_{x} u(x, T) \neq 0, \quad \text { for } x \in I_{j},
$$

and $u(\cdot, T)$ is constant outside of these intervals. Let $U_{j}$ be the range of $u(x, T)$ on $x \in I_{j}$. Then, on each $I_{j}$ we make the change of variables $x \in I_{j} \rightarrow y_{j}(x)=$ $u(x, T) \in U_{j}$, with inverse $y \in U_{j} \rightarrow x_{j}(y) \in I_{j}$. We have

$$
d x=\frac{d x_{j}}{d y} d y, \quad \partial_{x}=\frac{d y_{j}}{d x} \partial_{y}=\left(\frac{d x_{j}}{d y}\right)^{-1} \partial_{y}, \quad \text { on } x \in I_{j}
$$

and therefore,

$$
\begin{aligned}
\delta J(f)(\delta f) & =-T \int_{\mathbb{R}} \partial_{x}(\delta f(u(x, T)))\left(u(x, T)-u^{d}(x)\right) d x \\
& =-T \sum_{j=1}^{J} \int_{U_{j}} \delta f^{\prime}(y)\left(y-u^{d}\left(x_{j}(y)\right)\right) d y \\
& =-T \int_{\bigcup_{j=1}^{J} U_{j}} \delta f^{\prime}(y) \sum_{j=1}^{J} \chi_{U_{j}}(y)\left(y-u^{d}\left(x_{j}(y)\right)\right) d y
\end{aligned}
$$

where $\chi_{U_{j}}(y)$ is the characteristic function of the set $U_{j}$.

This expression provides a natural way to compute a descent direction for the continuous functional $J$. We just take $\delta f$ such that

$$
\delta f^{\prime}(y)=\sum_{j=1}^{J} \chi_{U_{j}}(y)\left(y-u^{d}\left(x_{j}(y)\right), \quad y \in \bigcup_{j=1}^{J} U_{j} .\right.
$$

Note that the value of $\delta f^{\prime}$ outside the set $\bigcup_{j=1}^{J} U_{j}$ does not affect the derivative of $J$. 
This yields the steepest descent direction for the continuous functional. Note, however, that this is not very useful since it requires a detailed analysis of the intervals where $u(x, T)$ is a monotone function. Moreover, in practice, it is more natural to deal with finite dimensional sets of admissible nonlinearities as in (4.5) rather than with the full complexity of the nonlinearities in (4.7).

Proof of Theorem 4.1. The first idea is to prove that, for $\varepsilon>0$ sufficiently small, the solution $u_{\varepsilon}(x, t)$ corresponding to the perturbed flux,

$$
f_{\varepsilon}(s)=f(s)+\varepsilon \delta f(s),
$$

is also a classical solution in $(x, t) \in \mathbb{R} \times[0, T]$ and it can be written as

$$
u_{\varepsilon}=u+\varepsilon \delta u+o(\varepsilon), \quad \text { with respect to the } C^{0} \text { topology, }
$$

where $\delta u$ is the solution of the linearized equation,

$$
\left\{\begin{array}{l}
\partial_{t} \delta u+\partial_{x}\left(f^{\prime}(u) \delta u\right)=-\partial_{x}(\delta f(u)) \\
\delta u(x, 0)=0
\end{array}\right.
$$

Assume for the moment that (4.9) holds. Now, let $\delta J$ be the Gateaux derivative of $J$ at $f$ in the direction $\delta f$. From (4.9) it is easy to see that

$$
\delta J=\int_{\mathbb{R}}\left(u(x, T)-u^{d}(x)\right) \delta u(x, T) d x,
$$

where $\delta u$ solves the linearized system above (4.10). Note that the solution of (4.10) is simply given by

$$
\delta u(x, t)=-t \partial_{x}(\delta f(u(x, t))) .
$$

In fact, it obviously, satisfies the initial condition $\delta u(x, 0)=0$ and

$$
\begin{aligned}
& \partial_{t}\left(-t \partial_{x}(\delta f(u(x, t)))\right)+\partial_{x}\left(-f^{\prime}(u) t \partial_{x}(\delta f(u(x, t)))\right) \\
& \quad=-\partial_{x}(\delta f(u(x, t)))-t \partial_{x}\left(\delta f^{\prime}(u(x, t)) \partial_{t} u\right)+t \partial_{x}\left(f^{\prime}(u(x, t)) \delta f^{\prime}(u(x, t)) \partial_{x} u\right) \\
& \quad=-\partial_{x}(\delta f(u(x, t)))-t \partial_{x}\left(\delta f^{\prime}(u(x, t))\left(\partial_{t} u-\partial_{x}(f(u))\right)=-\partial_{x}(\delta f(u(x, t))) .\right.
\end{aligned}
$$

Formula (4.3) is finally obtained substituting (4.12) into formula (4.11).

It remains to see that (4.9) holds. The proof is based on the classical characteristics approach by writing both $u$ and $u_{\varepsilon}$ in terms of their initial data. We need some previous estimates,

$$
\begin{aligned}
& \left\|u_{\varepsilon}(\cdot, t)\right\|_{\text {Lip }} \leq C, \\
& \frac{1}{\varepsilon}\left\|u_{\varepsilon}(\cdot, t)-u(\cdot, t)\right\|_{L^{\infty}(\mathbb{R})} \leq C, \\
& \lim _{\varepsilon \rightarrow 0}\left\|\partial_{x} u_{\varepsilon}(\cdot, t)-\partial_{x} u(\cdot, t)\right\|_{L^{\infty}(\mathbb{R})}=0,
\end{aligned}
$$

uniformly in $t \in[0, T]$, for some constant $C>0$. Here, $\left\|u_{\varepsilon}(\cdot, t)\right\|_{\text {Lip }}$ stands for the Lipschitz norm of $u_{\varepsilon}(\cdot, t)$.

Let us prove (4.13). Given $0 \leq t_{0}<t \leq T$ and $x, x^{\prime} \in \mathbb{R}$, we have

$$
\begin{aligned}
& \left|u_{\varepsilon}\left(x^{\prime}, t\right)-u_{\varepsilon}(x, t)\right| \\
& \quad=\left|u_{\varepsilon}\left(x^{\prime}-\left(t-t_{0}\right) f_{\varepsilon}^{\prime}\left(u_{\varepsilon}\left(x^{\prime}, t\right)\right), t_{0}\right)-u_{\varepsilon}\left(x-\left(t-t_{0}\right) f_{\varepsilon}^{\prime}\left(u_{\varepsilon}(x, t)\right), t_{0}\right)\right| \\
& \quad \leq\left\|u_{\varepsilon}\left(\cdot, t_{0}\right)\right\|_{L i p}\left(\left|x-x^{\prime}\right|+\left(t-t_{0}\right)\left|f_{\varepsilon}^{\prime}\left(u_{\varepsilon}\left(x^{\prime}, t\right)\right)-f_{\varepsilon}^{\prime}\left(u_{\varepsilon}(x, t)\right)\right|\right) \\
& \quad \leq\left\|u_{\varepsilon}\left(\cdot, t_{0}\right)\right\|_{L i p}\left(\left|x-x^{\prime}\right|+\left(t-t_{0}\right)\left\|f_{\varepsilon}^{\prime \prime}\right\|_{L^{\infty}}\left|u_{\varepsilon}\left(x^{\prime}, t\right)-u_{\varepsilon}(x, t)\right|\right),
\end{aligned}
$$

where $\left\|f_{\varepsilon}^{\prime \prime}\right\|_{L^{\infty}}$ is the norm of $f_{\varepsilon}^{\prime \prime}$ in $L^{\infty}\left(\left[-\left\|u^{0}\right\|_{L^{\infty}(\mathbb{R})},\left\|u^{0}\right\|_{L^{\infty}(\mathbb{R})}\right]\right)$ since, by the maximum principle, this interval contains the range of $u_{\varepsilon}(x, t)$ for all $x \in \mathbb{R}$ and 
$t \in[0, T]$. Obviously $\left\|f_{\varepsilon}^{\prime \prime}\right\|_{L^{\infty}}$ is uniformly bounded in $\varepsilon$ for any function $f_{\varepsilon}=f+\varepsilon \delta f$ with $f, \delta f \in C^{2}(\mathbb{R})$.

Thus, for $t-t_{0}<\left\|u_{\varepsilon}\left(\cdot, t_{0}\right)\right\|_{L i p}^{-1}\left\|f_{\varepsilon}^{\prime \prime}\right\|_{L^{\infty}}^{-1}$, we have

$$
\left|u_{\varepsilon}\left(x^{\prime}, t\right)-u_{\varepsilon}(x, t)\right| \leq \frac{\left\|u_{\varepsilon}\left(\cdot, t_{0}\right)\right\|_{L i p}}{1-\left(t-t_{0}\right)\left\|u_{\varepsilon}\left(\cdot, t_{0}\right)\right\|_{L i p}\left\|f_{\varepsilon}^{\prime \prime}\right\|_{L^{\infty}}}\left|x-x^{\prime}\right| .
$$

If we apply formula (4.16) with $t_{0}=0$ we obtain that the Lipschitz constant for $u_{\varepsilon}(\cdot, t)$ is uniform in $\varepsilon$ for $t \in\left[0, t_{1}\right]$ with $t_{1}<\left\|u^{0}\right\|_{L i p}^{-1}\left\|f_{\varepsilon}^{\prime \prime}\right\|_{L^{\infty}}^{-1}$, since $u_{\varepsilon}(x, 0)=u^{0}(x)$ which does not depend on $\varepsilon$. In particular, the Lipschitz constant for $u_{\varepsilon}\left(\cdot, t_{1}\right)$ is uniform in $\varepsilon$.

Note that the same argument with $t_{0}=t_{1}$ in formula (4.16) provides the analogous result for the larger time $t \in\left[0, t_{2}\right]$ where $t_{2}$ is such that $t_{2}-t_{1}<$ $\left\|u_{\varepsilon}\left(\cdot, t_{1}\right)\right\|_{L i p}^{-1}\left\|f_{\varepsilon}^{\prime \prime}\right\|_{L^{\infty}}^{-1}$. Following an iterative process we could expect to cover the whole time interval $[0, T]$. However, the Lipschitz bound for $u_{\varepsilon}\left(\cdot, t_{n}\right)$ may possibly deteriorate in time and this argument could fail to reach the final time $T$, since in this case

$$
t_{n+1}-t_{n}<\left\|u_{\varepsilon}\left(\cdot, t_{n}\right)\right\|_{L i p}^{-1}\left\|f_{\varepsilon}^{\prime \prime}\right\|_{L^{\infty}}^{-1} \rightarrow 0, \quad \text { as } n \rightarrow \infty .
$$

Thus, before considering larger times, we prove that the Lipschitz constant for $u_{\varepsilon}\left(\cdot, t_{1}\right)$ can be shown to be independent of $t \in\left[0, t_{1}\right]$. In this way, the right-hand side in (4.17) is uniformly bounded from below and the sequence $t_{n}$ can be chosen in such a way that the iterative argument above will cover the whole interval $[0, T]$.

The idea is to prove that (4.14) and (4.15) hold uniformly in $t \in\left[0, t_{1}\right]$. Then, the uniform bound for the Lipschitz norm of $u_{\varepsilon}(\cdot, t)$ in $t \in\left[0, t_{1}\right]$ is easily obtained from the regularity of $u(x, t)$ and the uniform convergence (4.15), using a perturbation argument that we show below.

Now, we prove that formulas (4.14) and (4.15) hold in $t \in\left[0, t_{1}\right]$. They are obtained with similar arguments. We focus on (4.15) since the proof of (4.14) is easier.

Given $t>t_{0} \geq 0$, we define $y(x, t)=x-\left(t-t_{0}\right) f^{\prime}(u(x, t))$ and $y_{\varepsilon}(x, t)=$ $x-\left(t-t_{0}\right) f_{\varepsilon}^{\prime}\left(u_{\varepsilon}(x, t)\right)$. We have

$$
\begin{aligned}
& \left|\partial_{x} u_{\varepsilon}(x, t)-\partial_{x} u(x, t)\right| \\
& =\left|\partial_{x}\left(u_{\varepsilon}\left(y_{\varepsilon}(x, t), t_{0}\right)\right)-\partial_{x}\left(u\left(y(x, t), t_{0}\right)\right)\right| \\
& \leq\left|\partial_{x} u_{\varepsilon}\left(y_{\varepsilon}(x, t), t_{0}\right)\right|\left(t-t_{0}\right)\left|f^{\prime \prime}\left(u_{\varepsilon}\right) \partial_{x} u_{\varepsilon}+\varepsilon \delta f^{\prime \prime}\left(u_{\varepsilon}\right) \partial_{x} u_{\varepsilon}-f^{\prime \prime}(u) \partial_{x} u\right| \\
& +\left|\partial_{x} u_{\varepsilon}\left(y_{\varepsilon}(x, t), t_{0}\right)-\partial_{x} u\left(y(x, t), t_{0}\right)\right|\left|1-\left(t-t_{0}\right) f^{\prime \prime}(u) \partial_{x} u\right| \\
& \leq\left(\varepsilon\left\|\delta f^{\prime \prime}\right\|_{L^{\infty}}+\left\|f^{\prime \prime}\left(u_{\varepsilon}(\cdot, t)\right) \partial_{x} u_{\varepsilon}-f^{\prime \prime}(u(\cdot, t)) \partial_{x} u\right\|_{L^{\infty}}\right)\left\|u_{\varepsilon}(\cdot, t)\right\|_{L i p}\left(t-t_{0}\right) \\
& +C\left|\partial_{x} u_{\varepsilon}\left(y_{\varepsilon}(x, t), t_{0}\right)-\partial_{x} u\left(y_{\varepsilon}(x, t), t_{0}\right)\right|+C\left|\partial_{x} u\left(y_{\varepsilon}(x, t), t_{0}\right)-\partial_{x} u\left(y(x, t), t_{0}\right)\right| \\
& \leq\left(\varepsilon\left\|\delta f^{\prime \prime}\right\|_{L^{\infty}}+\left\|f^{\prime \prime}\right\|_{L^{\infty}}\left\|\partial_{x} u_{\varepsilon}(\cdot, t)-\partial_{x} u(\cdot, t)\right\|_{L^{\infty}}\right)\left\|u_{\varepsilon}(\cdot, t)\right\|_{L i p}\left(t-t_{0}\right) \\
& +\left(\left\|f^{\prime \prime}\left(u_{\varepsilon}(\cdot, t)\right)-f^{\prime \prime}(u(\cdot, t))\right\|_{L^{\infty}}\|u\|_{L i p}\right)\left\|u_{\varepsilon}\right\|_{L i p}\left(t-t_{0}\right) \\
& +C\left\|\partial_{x} u_{\varepsilon}\left(\cdot, t_{0}\right)-\partial_{x} u\left(\cdot, t_{0}\right)\right\|_{L^{\infty}}+C\left|\partial_{x} u\left(y_{\varepsilon}(x, t), t_{0}\right)-\partial_{x} u\left(y(x, t), t_{0}\right)\right|,
\end{aligned}
$$

with $C=1+\left(t-t_{0}\right)\left\|f^{\prime \prime}\right\|_{L^{\infty}}\|u\|_{L i p}$. Thus, if we choose

$$
\left(t-t_{0}\right)<\frac{1}{2} \min _{0<\varepsilon<\varepsilon_{0}}\left\|u_{\varepsilon}\right\|_{L i p}^{-1}\left\|f^{\prime \prime}\right\|_{L^{\infty}}^{-1},
$$


then

$$
\begin{aligned}
& \frac{1}{2}\left\|\partial_{x} u_{\varepsilon}(\cdot, t)-\partial_{x} u(\cdot, t)\right\|_{L^{\infty}} \leq \varepsilon\left\|\delta f^{\prime \prime}\right\|_{L^{\infty}}\left\|u_{\varepsilon}(\cdot, t)\right\|_{L i p}\left(t-t_{0}\right) \\
& \quad+\left\|f^{\prime \prime}\left(u_{\varepsilon}(\cdot, t)\right)-f^{\prime \prime}(u(\cdot, t))\right\|_{L^{\infty}}\|u\|_{L i p}\left\|u_{\varepsilon}\right\|_{L i p}\left(t-t_{0}\right) \\
& \quad+C\left|\partial_{x} u\left(x-\left(t-t_{0}\right) f_{\varepsilon}^{\prime}\left(u_{\varepsilon}\right), t_{0}\right)-\partial_{x} u\left(x-\left(t-t_{0}\right) f^{\prime}(u), t_{0}\right)\right| \\
& \quad+C\left\|\partial_{x} u_{\varepsilon}\left(\cdot, t_{0}\right)-\partial_{x} u\left(x, t_{0}\right)\right\|_{L^{\infty}} .
\end{aligned}
$$

Now we take the limit as $\varepsilon \rightarrow 0$ in this expression. The first term on the righthand side converges to zero from the uniform bound of $\left\|u_{\varepsilon}\right\|_{L i p}$. For the second term we also use the regularity of $f \in C^{2}$, and the fact that we are assuming the uniform convergence $\left\|u_{\varepsilon}(\cdot, t)-u(\cdot, t)\right\|_{L^{\infty}(\mathbb{R})} \rightarrow 0$, as $\varepsilon \rightarrow 0$, stated in (4.14). The convergence of the third term is easily obtained from the uniform continuity of $\partial_{x} u$. In fact, $u \in C_{0}^{1}$ since it is a classical solution with $u_{0} \in C_{0}^{1}(\mathbb{R})$. Finally, the last limit goes to zero if $t_{0}=0$. This allows us to prove the convergence of the right-hand side, uniformly in $t \in\left[0, t_{1}\right]$ with $t_{1}=\min _{0<\varepsilon<\varepsilon_{0}}\left\|u_{\varepsilon}\right\|_{\text {Lip }}^{-1}\left\|f^{\prime \prime}\right\|_{L^{\infty}}^{-1}$.

Once we have proved (4.15) uniformly in $t \in\left[0, t_{1}\right]$ we can deduce a uniform bound for the Lipschitz constant of $u_{\varepsilon}$, independent of $t \in\left[0, t_{1}\right]$. As we said above, we use a perturbation argument,

$$
\left\|\partial_{x} u_{\varepsilon}(\cdot, t)\right\|_{L^{\infty}} \leq\left\|\partial_{x} u(\cdot, t)\right\|_{L^{\infty}}+\left\|\partial_{x} u_{\varepsilon}(\cdot, t)-\partial_{x} u(\cdot, t)\right\|_{L^{\infty}} .
$$

The hypotheses on the initial datum $u^{0}$ and $T$ guarantee that the solution $u \in$ $C_{0}^{1}(\mathbb{R} \times[0, T])$ and therefore $\left\|\partial_{x} u(\cdot, t)\right\|_{L^{\infty}}$ can be bounded by a constant $C$ independent of $t \in[0, T]$. On the other hand, the convergence (4.15) guarantees that we can choose $\varepsilon>0$ sufficiently small so that the second term in (4.19) is also bounded by a constant independent of $t \in\left[0, t_{1}\right]$.

Estimate (4.13) for larger time is obtained from a continuation argument. We choose $t_{2}$ such that $t_{2}-t_{1}<\left\|u_{\varepsilon}\left(\cdot, t_{1}\right)\right\|_{L i p}^{-1}\left\|f_{\varepsilon}^{\prime \prime}\right\|_{L^{\infty}}^{-1}$. From formula (4.16) we obtain a bound of the Lipschitz norm of $u_{\varepsilon}(\cdot, t)$, uniform in $\varepsilon$, for $t \in\left[0, t_{2}\right]$. This allows us to prove (4.14) and (4.15) in $t \in\left[0, t_{2}\right]$, and a bound for the Lipschitz norm of $u_{\varepsilon}$ independent of $t \in\left[0, t_{2}\right]$. Following an iterative process we obtain a similar result on $t \in\left[0, t_{n}\right], n \geq 1$. Note that we cover the interval $[0, T]$ in a finite number of steps since the bound for $t_{n+1}-t_{n}$, given by $\left\|u_{\varepsilon}\left(\cdot, t_{n}\right)\right\|_{L i p}^{-1}\left\|f_{\varepsilon}^{\prime \prime}\right\|_{L^{\infty}}^{-1}$, is bounded below by a constant independent of $t_{n}$.

Once the previous estimates (4.13)-(4.15) are obtained we go back to the proof of (4.9). Let us write

$$
w_{\varepsilon}=\frac{1}{\varepsilon}\left(u_{\varepsilon}-u-\varepsilon \delta u\right) .
$$

We have to see that $\lim _{\varepsilon \rightarrow 0}\left\|u_{\varepsilon}(\cdot, t)\right\|_{L^{\infty}(\mathbb{R})}=0$ uniformly in $t \in[0, T]$. The function $w_{\varepsilon}$ satisfies system

$$
\left\{\begin{array}{cl}
\partial_{t} w_{\varepsilon}+\partial_{x}\left(f^{\prime}(u) w_{\varepsilon}\right)=-\partial_{x}\left(\delta f\left(u_{\varepsilon}\right)-\delta f(u)\right) & \\
-\frac{1}{\varepsilon} \partial_{x}\left(f\left(u_{\varepsilon}\right)-f(u)-f^{\prime}(u)\left(u_{\varepsilon}-u\right)\right), & t \in[0, T], x \in \mathbb{R}, \\
w_{\varepsilon}(x, 0)=0, & x \in \mathbb{R} .
\end{array}\right.
$$

Note that system (4.20) is a linear conservation law where the nonhomogeneous term is small, for $\varepsilon$ small. System (4.20) is analogous to the linearized system (4.10) for which the solution is given by (4.12). Thus, the solution of (4.20) is given by,

$$
w_{\varepsilon}=-t \partial_{x}\left(\delta f\left(u_{\varepsilon}\right)-\delta f(u)\right)-\frac{t}{\varepsilon} \partial_{x}\left(f\left(u_{\varepsilon}\right)-f(u)-f^{\prime}(u)\left(u_{\varepsilon}-u\right)\right) .
$$


Then,

$$
\begin{aligned}
& \left|w_{\varepsilon}(x, t)\right| \leq t\left|\delta f^{\prime}\left(u_{\varepsilon}\right) \partial_{x} u_{\varepsilon}-\delta f^{\prime}(u) \partial_{x} u\right| \\
& +\frac{t}{\varepsilon}\left|f^{\prime}\left(u_{\varepsilon}\right) \partial_{x} u_{\varepsilon}-f^{\prime}(u) \partial_{x} u-f^{\prime \prime}(u) \partial_{x} u\left(u_{\varepsilon}-u\right)-f^{\prime}(u)\left(\partial_{x} u_{\varepsilon}-\partial_{x} u\right)\right| \\
& \leq t\left|\delta f^{\prime}\left(u_{\varepsilon}\right)-\delta f^{\prime}(u)\right|\left|\partial_{x} u\right|+t\left|\delta f^{\prime}(u)\right|\left|\partial_{x} u_{\varepsilon}-\partial_{x} u\right| \\
& +\frac{t}{\varepsilon}\left|f^{\prime}\left(u_{\varepsilon}\right)-f^{\prime}(u)-f^{\prime \prime}(u)\left(u_{\varepsilon}-u\right)\right|\left|\partial_{x} u\right| \\
& +\frac{t}{\varepsilon}\left|f^{\prime}\left(u_{\varepsilon}\right)-f^{\prime}(u)\right|\left|\partial_{x} u_{\varepsilon}-\partial_{x} u\right| \\
& \leq t\left\|\delta f^{\prime \prime}\right\|_{L^{\infty}}\left\|u_{\varepsilon}-u\right\|_{L^{\infty}}\|u\|_{L i p}+t\left\|\delta f^{\prime}\right\|_{L^{\infty}}\left\|\partial_{x} u_{\varepsilon}-\partial_{x} u\right\|_{L^{\infty}} \\
& +\frac{t}{\varepsilon}\left\|\partial_{x} u\right\|_{L^{\infty}}\left\|f^{\prime}\left(u_{\varepsilon}\right)-f^{\prime}(u)-f^{\prime \prime}(u)\left(u_{\varepsilon}-u\right)\right\|_{L^{\infty}} \\
& +\frac{t}{\varepsilon}\left\|f^{\prime \prime}\right\|_{L^{\infty}}\left\|u_{\varepsilon}-u\right\|_{L^{\infty}}\left\|\partial_{x} u_{\varepsilon}-\partial_{x} u\right\|_{L^{\infty}}
\end{aligned}
$$

which converges to zero, as $\varepsilon \rightarrow 0$, in view of (4.13)-(4.15) and the regularity of $f$.

Remark 4.4. Formula (4.12) for $\delta u(x, T)$ can be obtained by the method of characteristics. In fact, (4.10) can be reduced to an ordinary differential equation when looking at the solution $\delta u$ along their characteristic curves, parametrized by $(x(t), t)$ with $t \in[0, T]$. In this way, $\delta u(x(t), t)$ satisfies

$$
\delta u(x(t), t)=-\int_{0}^{t} \exp \left(-\int_{s}^{t} \partial_{x}\left(f^{\prime}(u)\right)(x(r), r) d r\right) \partial_{x}(\delta f(u))(x(s), s) d s .
$$

This expression can be simplified by using the biparametric family of changes of variables $y_{s, t}: \mathbb{R} \rightarrow \mathbb{R}$ defined by,

$$
y_{s, t}(x)=x+f^{\prime}(u(x, s))(t-s), \quad t \in[0, T], \text { and } s \in[0, t] .
$$

Note that, for fixed $x \in \mathbb{R}$ and $s \in[0, T]$, the function $r \rightarrow\left(x+f^{\prime}(u(x, s)) r, s+r\right)$ with $r \in(-t, T-t)$ is a parametrization of the characteristic line, associated to (1.1), which contains the point $(x, s)$. Thus, the point of coordinates $\left(y_{s, t}(x), t\right)$ is, in fact, the point of this characteristic line corresponding to the time $t$, i.e., $r=t-s$. In particular,

$$
y_{s, t}(x(s))=x(t), \quad \text { for all } 0 \leq s \leq t \leq T .
$$

This provides an easy geometric interpretation of these changes of variables that are well-defined since we are assuming that $u$ does not generate shocks in $t \in(0, T)$. If we apply the change of variables $x \rightarrow y_{r, t}(x)$ for $\partial_{x}\left(f^{\prime}(u(x, t))\right)$ and $x \rightarrow y_{s, t}(x)$ for $\partial_{x}(\delta f(u(x, t)))$, we see that (4.21) and (4.12) are equal.

4.2. Linearization in the presence of shocks. In this section we collect some existing results on the sensitivity of the solution of conservation laws in the presence of shocks. We follow the analysis in 9 but similar results in different forms and degrees of generality can be found in [1], 4], [5], 35] or [19, for example.

We focus on the particular case of solutions having a single shock in a finite time interval $t \in[0, T]$, but the analysis can be extended to consider more general onedimensional systems of conservation laws with a finite number of noninteracting shocks. The theory of duality and reversible solutions developed in [4] and [5] is the one leading to more general results. 
We introduce the following hypothesis:

$(\mathrm{H})$ Assume that $u(x, t) \in B V(\mathbb{R} \times(0, T)) \cap C^{0}\left([0, T] ; L_{l o c}^{1}(\mathbb{R})\right)$ is a weak entropy solution of (1.1) with a single discontinuity along a regular curve $\Sigma=$ $\{(t, \varphi(t)), t \in[0, T+\tau]\}$, for some $\tau>0$, which is uniformly Lipschitz continuous outside $\Sigma$. In particular, it satisfies the Rankine-Hugoniot condition on $\Sigma$ :

$$
\varphi^{\prime}(t)[u]_{\varphi(t)}=[f(u)]_{\varphi(t)}, \quad 0 \leq t \leq T+\tau .
$$

Here we have used the notation $[v]_{x_{0}}=v\left(x_{0}^{+}\right)-v\left(x_{0}^{-}\right)$for the jump at $x_{0}$ of any piecewise continuous function $v$ with a discontinuity at $x=x_{0}$.

Note that hypothesis $(\mathrm{H})$ above concerns a time interval $[0, T+\tau]$ larger than expected for our optimization problem, which only requires the value of the solution $u$ at time $t=T$. This is to guarantee that, for solutions of (1.1) satisfying $(\mathrm{H})$, sufficiently small perturbations of the flux function $f$ in (1.1) will provide solutions which still satisfy hypothesis $(\mathrm{H})$, with possibly different values of $\tau>0$.

Note that $\Sigma$ divides $\mathbb{R} \times(0, T)$ in two parts: $Q^{-}$and $Q^{+}$, the subdomains of $\mathbb{R} \times(0, T)$ to the left and to the right of $\Sigma$ respectively.

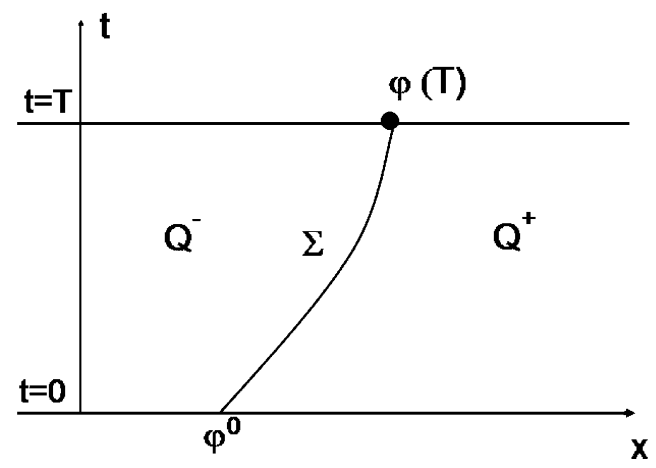

Figure 1. Subdomains $Q^{-}$and $Q^{+}$.

As we will see, in the presence of shocks, for correctly dealing with optimal control and design problems, the state of the system has to be viewed as being a pair $(u, \varphi)$ combining the solution of (1.1) and the shock location $\varphi$. This is relevant in the analysis of sensitivity of functions below and when applying descent algorithms.

Then the pair $(u, \varphi)$ satisfies the system

$$
\begin{cases}\partial_{t} u+\partial_{x}(f(u))=0, & \text { in } Q^{-} \cup Q^{+}, \\ \varphi^{\prime}(t)[u]_{\varphi(t)}=[f(u)]_{\varphi(t)}, & t \in(0, T), \\ \varphi(0)=\varphi^{0}, & \\ u(x, 0)=u^{0}(x), & \text { in }\left\{x<\varphi^{0}\right\} \cup\left\{x>\varphi^{0}\right\} .\end{cases}
$$

We now analyze the sensitivity of $(u, \varphi)$ with respect to perturbations of the flux $f$. To be precise, we adopt the functional framework based on the generalized tangent vectors introduced in [9].

Definition 4.1 ([9]). Let $v: \mathbb{R} \rightarrow \mathbb{R}$ be a piecewise Lipschitz continuous function with a single discontinuity at $y \in \mathbb{R}$. We define the set $\Gamma_{(v, y)}$ as the family of all continuous paths $\gamma:\left[0, \varepsilon_{0}\right] \rightarrow L^{1}(\mathbb{R}) \times \mathbb{R}$ with 
1. $\gamma(0)=(v, y)$ and $\varepsilon_{0}>0$ possibly depending on $\gamma$.

2. For any $\varepsilon \in\left[0, \varepsilon_{0}\right], \gamma(\varepsilon)=\left(v_{\varepsilon}, y_{\varepsilon}\right)$ where the functions $v_{\varepsilon}$ are piecewise Lipschitz with a single discontinuity at $x=y_{\varepsilon}$, depending continuously on $\varepsilon$, and there exists a constant $L$ independent of $\varepsilon \in\left[0, \varepsilon_{0}\right]$ such that

$$
\left|v_{\varepsilon}(x)-v_{\varepsilon}\left(x^{\prime}\right)\right| \leq L\left|x-x^{\prime}\right|,
$$

whenever $y_{\varepsilon} \notin\left[x, x^{\prime}\right]$.

The paths $\gamma(\varepsilon)=\left(v_{\varepsilon}, y_{\varepsilon}\right) \in \Gamma_{(v, y)}$ will be referred to as regular variations of $(v, y)$.

Furthermore, we define the set $T_{(v, y)}$ of generalized tangent vectors of $(v, y)$ as the space of $(\delta v, \delta y) \in L^{1} \times \mathbb{R}$ for which the path $\gamma_{(\delta v, \delta y)}$ given by $\gamma_{(\delta v, \delta y)}(\varepsilon)=\left(v_{\varepsilon}, y_{\varepsilon}\right)$ with

$$
v_{\varepsilon}=\left\{\begin{array}{l}
v+\varepsilon \delta v+[v]_{y} \chi_{[y+\varepsilon \delta y, y]} \text { if } \delta y<0, \\
v+\varepsilon \delta v-[v]_{y} \chi_{[y, y+\varepsilon \delta y]} \text { if } \delta y>0,
\end{array} \quad y_{\varepsilon}=y+\varepsilon \delta y\right.
$$

satisfies $\gamma_{(\delta v, \delta y)} \in \Gamma_{(v, y)}$.

Finally, we define the equivalence relation $\sim$ defined on $\Gamma_{(v, y)}$ by

$$
\gamma \sim \gamma^{\prime} \text { if and only if } \lim _{\varepsilon \rightarrow 0} \frac{\left\|v_{\varepsilon}-v_{\varepsilon}^{\prime}\right\|_{L^{1}}}{\varepsilon}=0,
$$

and we say that a path $\gamma \in \Gamma_{(v, y)}$ generates the generalized tangent vector $(\delta v, \delta y) \in$ $T_{(v, y)}$ if $\gamma$ is equivalent to $\gamma_{(\delta v, \delta y)}$ as in (4.26).

Remark 4.5. The path $\gamma_{(\delta v, \delta y)} \in \Gamma_{(v, y)}$ in (4.26) represents, at first order, the variation of a function $v$ by adding a perturbation function $\varepsilon \delta v$ and by shifting the discontinuity by $\varepsilon \delta y$.

Note that, for a given pair $(v, y)$ ( $v$ being a piecewise Lipschitz continuous function with a single discontinuity at $y \in \mathbb{R}$ ) the associated generalized tangent vectors $(\delta v, \delta y) \in T_{(v, y)}$ are those pairs for which $\delta v$ is Lipschitz continuous with a single discontinuity at $x=y$.

The main result in this section is the following:

Theorem 4.2. Let $f \in C^{2}(\mathbb{R})$ and $(u, \varphi)$ be a solution of (4.25) satisfying hypothesis (H) in (4.24). Consider a variation $\delta f \in C^{2}(\mathbb{R})$ for $f$ and let $f_{\varepsilon}=f+\varepsilon \delta f \in$ $C^{2}(\mathbb{R})$ be a new flux obtained by an increment of $f$ in the direction $\delta f$. Assume that $\left(u_{\varepsilon}, \varphi_{\varepsilon}\right)$ is the unique entropy solution of (4.25) with flux $f_{\varepsilon}$. Then, for all $t \in[0, T]$ the path $\left(u_{\varepsilon}(\cdot, t), \varphi_{\varepsilon}(t)\right)$ is a regular variation for $(u(\cdot, t), \varphi(t))$ (in the sense of Definition 4.1) generating a tangent vector $(\delta u(\cdot, t), \delta \varphi(t)) \in L^{1} \times \mathbb{R}$, i.e., $\left(u_{\varepsilon}(\cdot, t), \varphi_{\varepsilon}(t)\right) \in \Gamma_{(\delta u(\cdot, t), \delta \varphi(t))}$. Moreover, $(\delta u, \delta \varphi) \in C\left([0, T] ;\left(L^{1}(\mathbb{R}) \times \mathbb{R}\right)\right)$ is the unique solution of the system

$$
\left\{\begin{array}{l}
\partial_{t} \delta u+\partial_{x}\left(f^{\prime}(u) \delta u\right)=-\partial_{x}(\delta f(u)), \quad \text { in } Q^{-} \cup Q^{+}, \\
\delta \varphi^{\prime}(t)[u]_{\varphi(t)}+\delta \varphi(t)\left(\varphi^{\prime}(t)\left[u_{x}\right]_{\varphi(t)}-\left[u_{x} f^{\prime}(u)\right]_{\varphi(t)}\right) \\
\quad+\varphi^{\prime}(t)[\delta u]_{\varphi(t)}-\left[f^{\prime}(u) \delta u\right]_{\varphi(t)}-[\delta f(u)]_{\varphi(t)}=0, \quad \text { in }(0, T), \\
\delta u(x, 0)=0, \quad \text { in }\left\{x<\varphi^{0}\right\} \cup\left\{x>\varphi^{0}\right\}, \\
\delta \varphi(0)=0 .
\end{array}\right.
$$

We prove this technical result in the Appendix at the end of the paper.

Remark 4.6. The linearized system (4.27) has a unique solution which can be computed in two steps. The method of characteristics determines $\delta u$ in $Q^{-} \cup Q^{+}$, i.e., 
outside $\Sigma$, from the initial data $\delta u^{0}$ (note that system (4.27) has the same characteristics as (4.25) ). We also have the value of $u$ and $u_{x}$ to both sides of the shock $\Sigma$ and this allows us to determine the coefficients of the ODE that $\delta \varphi$ satisfies. Then, we solve the ordinary differential equation to obtain $\delta \varphi$.

Remark 4.7. Due to the finite speed of propagation for (1.1) the result in Theorem 4.2 can be stated locally, i.e., in a neighborhood of the shock $\Sigma$. Thus, Theorem 4.2 can be easily generalized for solutions having a finite number of noninteracting shocks.

Remark 4.8. The equation for $\delta \varphi$ in (4.27) is formally obtained by linearizing the Rankine-Hugoniot condition $[u] \varphi^{\prime}(t)=[f(u)]$ with the formula $\delta[u]=[\delta u]+\left[u_{x}\right] \delta \varphi$.

We finish this section with the following result which characterizes the solutions of (4.27).

Theorem 4.3. Let $f \in C^{2}(\mathbb{R})$ and $(u, \varphi)$ be a solution of (4.25) satisfying hypothesis $(H)$ in (4.24). The solution $(\delta u, \delta \varphi) \in C\left([0, T] ;\left(L^{1}(\mathbb{R}) \times \mathbb{R}\right)\right)$ of the system (4.27) is given by,

$$
\begin{aligned}
& \delta u(x, t)=-t \partial_{x}(\delta f(u(x, t))), \quad \text { in } Q^{-} \cup Q^{+}, \\
& \delta \varphi(t)=t \frac{[\delta f(u(\cdot, t))]_{\varphi(t)}}{[u(\cdot, t)]_{\varphi(t)}}, \quad \text { for all } t \in[0, T] .
\end{aligned}
$$

Proof. Formula (4.28) holds when $u$ is smooth, as we stated in (4.12). The same formula holds in the region where $u$ is Lipschitz, i.e., $Q^{-} \cup Q^{+}$, by a density argument.

We now prove formula (4.29). Without loss of generality, we focus on the case $t=T$. For simplicity we divide this part of the proof into two steps. First we show that

$$
T[\delta f(u(\cdot, T))]_{\varphi(T)}=\int_{\Sigma}\left([\delta u],\left[f^{\prime}(u) \delta u+\delta f(u)\right]\right) \cdot \mathbf{n} d \Sigma
$$

and then,

$$
\int_{\Sigma}\left([\delta u],\left[f^{\prime}(u) \delta u+\delta f(u)\right]\right) \cdot \mathbf{n} d \Sigma=\delta \varphi(T)[u(\cdot, T)]_{\varphi(T)} .
$$

At this point we have to introduce some notation. Let

$$
x^{-}=\varphi(T)-f^{\prime}\left(u\left(\varphi(T)^{-}, T\right)\right) T, \quad x^{+}=\varphi(T)-f^{\prime}\left(u\left(\varphi(T)^{+}, T\right)\right) T,
$$

and consider the following subsets (see Figure 2),

$$
\begin{aligned}
& \hat{Q}^{-}=\left\{(x, t) \in \mathbb{R} \times(0, T) \text { such that } x<\varphi(T)-f^{\prime}\left(u\left(\varphi(T)^{-}, T\right)\right)(T-t)\right\} \\
& \hat{Q}^{+}=\left\{(x, t) \in \mathbb{R} \times(0, T) \text { such that } x>\varphi(T)-f^{\prime}\left(u\left(\varphi(T)^{+}, T\right)\right)(T-t)\right\} \\
& \mathcal{D}^{-}=Q^{-} \backslash \hat{Q}^{-}, \quad \mathcal{D}^{+}=Q^{+} \backslash \hat{Q}^{-}
\end{aligned}
$$

Note that, in $\hat{Q}^{-} \cup \hat{Q}^{+}$the characteristics do not meet the shock at any time $t \in[0, T]$.

In order to prove (4.30) we write the first equation in (4.27) as

$$
\operatorname{div}_{t, x}\left(\delta u, f^{\prime}(u) \delta u+\delta f(u)\right)=0, \quad \text { in } Q^{-} \cup Q^{+} .
$$




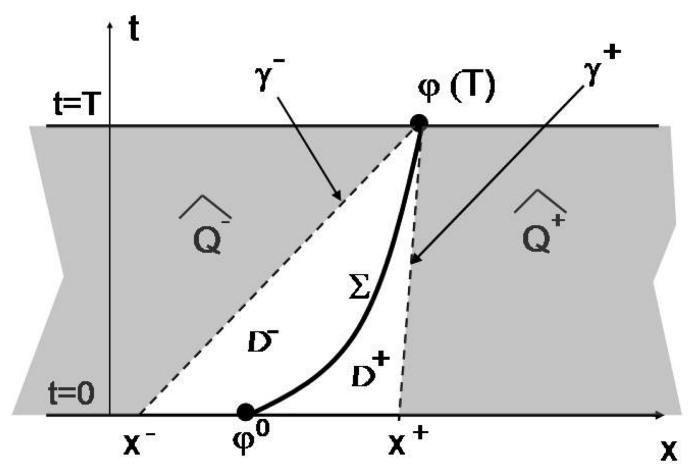

FiguRE 2. Subdomains $\hat{Q}^{-}$and $\hat{Q}^{+}$

Thus, integrating this equation over the triangle $\mathcal{D}^{-}=Q^{-} \backslash \hat{Q}^{-}$, using the divergence theorem and the fact that $\delta u(x, 0)=\delta u^{0}(x)=0$ for all $x \in \mathbb{R}$, we obtain

$$
\begin{aligned}
0= & \int_{\partial \mathcal{D}^{-}}\left(\delta u, f^{\prime}(u) \delta u+\delta f(u)\right) \cdot \mathbf{n} d \sigma=\int_{\Sigma}\left(\delta u^{-}, f^{\prime}\left(u^{-}\right) \delta u^{-}+\delta f\left(u^{-}\right)\right) \cdot \mathbf{n} d \Sigma \\
(4.36) \quad & +\int_{\gamma^{-}}\left(\delta u, f^{\prime}(u) \delta u+\delta f(u)\right) \cdot \mathbf{n} d \sigma,
\end{aligned}
$$

where $\mathbf{n}$ denotes the normal exterior vector to the boundary of $\mathcal{D}^{-}$and, for any continuous function $v$, defined in $x \in Q^{-}$, we have noted that

$$
v^{-}(x)=\lim _{\varepsilon \rightarrow 0} v(x-\varepsilon \mathbf{n}), \quad \text { for } x \in \Sigma .
$$

The curve $\gamma^{-}$in (4.36) is the characteristic line joining $(t, x)=\left(0, x^{-}\right)$with $(t, x)=$ $(T, \varphi(T))$. A parametrization of this line is given by

$$
\gamma^{-}:\left\{\left(t, \gamma^{-}(t)\right) \in(0, T) \times \mathbb{R} \text { such that } \gamma^{-}(t)=x^{-}+t f^{\prime}\left(u\left(x^{-}, 0\right)\right), t \in(0, T)\right\} .
$$

With this parametrization the last integral in the right-hand side of (4.36) can be written as

$$
\int_{\gamma^{-}}\left(\delta u, f^{\prime}(u) \delta u+\delta f(u)\right) \cdot \mathbf{n} d \sigma=\int_{0}^{T} \delta f\left(u\left(\gamma^{-}(t), t\right)\right) d t=T \delta f\left(u\left(\varphi(T)^{-}, T\right)\right),
$$

where the last identity comes from the fact that $u$ is constant along $\gamma^{-}$, i.e., $u\left(\gamma^{-}(t), t\right)=u\left(\varphi(T)^{-}, T\right)$ for all $t \in[0, T]$.

Thus, taking into account (4.38), the identity (4.36) reads

$$
0=\int_{\Sigma}\left(\delta u^{-}, f^{\prime}\left(u^{-}\right) \delta u^{-}+\delta f\left(u^{-}\right)\right) \cdot \mathbf{n} d \Sigma+T \delta f\left(u\left(\varphi(T)^{-}, T\right)\right)
$$

Of course we obtain a similar formula if we integrate (4.35) this time over $\mathcal{D}^{+}=$ $Q^{+} \backslash \hat{Q}^{+}$,

$$
0=-\int_{\Sigma}\left(\delta u^{+}, f^{\prime}\left(u^{+}\right) \delta u^{+}+\delta f\left(u^{+}\right)\right) \cdot \mathbf{n} d \Sigma-T \delta f\left(u\left(\varphi(T)^{+}, T\right)\right)
$$

The sign in (4.40) is changed with respect to (4.39) since we maintain the same choice for the normal vector.

Combining the identities (4.39) and (4.40) we easily obtain (4.30). 
Finally, we prove that (4.31) holds true. We consider the following parametrization of $\Sigma$ :

$$
\Sigma=\{(x, t) \in \mathbb{R} \times(0, T), \text { such that } x=\varphi(t), \text { with } t \in(0, T)\} .
$$

The cartesian components of the normal vector to $\Sigma$ at the point $(x, t)=(\varphi(t), t)$ are given by

$$
n_{t}=\frac{-\varphi^{\prime}(t)}{\sqrt{1+\left(\varphi^{\prime}(t)\right)^{2}}}, \quad n_{x}=\frac{1}{\sqrt{1+\left(\varphi^{\prime}(t)\right)^{2}}} .
$$

This, together with the second equation in system (4.27), give

$$
\begin{aligned}
& {[\delta u]_{\Sigma} n_{t}+\left[f^{\prime}(u) \delta u+\delta f(u)\right]_{\Sigma} n_{x}} \\
& =\frac{-\varphi^{\prime}(t)[\delta u(\cdot, t)]_{\varphi(t)}+\left[f^{\prime}(u(\cdot, t)) \delta u(\cdot, t)+\delta f(u(\cdot, t))\right]_{\varphi(t)}}{\sqrt{1+\left(\varphi^{\prime}(t)\right)^{2}}} \\
& \quad=\frac{\delta \varphi^{\prime}(t)[u(\cdot, t)]_{\varphi(t)}+\delta \varphi(t)\left(\varphi^{\prime}(t)\left[\partial_{x} u(\cdot, t)\right]_{\varphi(t)}-\left[\partial_{x}(f(u))(\cdot, t)\right]_{\varphi(t)}\right)}{\sqrt{1+\left(\varphi^{\prime}(t)\right)^{2}}} \\
& =\frac{\delta \varphi^{\prime}(t)[u(\cdot, t)]_{\varphi(t)}+\delta \varphi(t)\left(\varphi^{\prime}(t)\left[\partial_{x} u(\cdot, t)\right]_{\varphi(t)}+\left[\partial_{t} u(\cdot, t)\right]_{\varphi(t)}\right)}{\sqrt{1+\left(\varphi^{\prime}(t)\right)^{2}}} \\
& =\frac{1}{\sqrt{1+\left(\varphi^{\prime}(t)\right)^{2}}} \frac{d}{d t}\left(\delta \varphi(t)[u(\cdot, t)]_{\varphi(t)}\right) .
\end{aligned}
$$

Thus, integrating on $\Sigma$,

$$
\begin{aligned}
& \int_{\Sigma}\left([\delta u]_{\Sigma} n_{t}+\left[f^{\prime}(u) \delta u+\delta f(u)\right]_{\Sigma} n_{x}\right) d \Sigma=\int_{0}^{T} \frac{d}{d t}\left(\delta \varphi(t)[u(\cdot, t)]_{\varphi(t)}\right) d t \\
& \quad=\delta \varphi(T)[u(\cdot, T)]_{\varphi(T)}-\delta \varphi(0)[u(\cdot, 0)]_{\varphi(0)}=\delta \varphi(T)[u(\cdot, t)]_{\varphi(T)},
\end{aligned}
$$

where the last identity is due to the initial condition $\delta \varphi(0)=0$ in (4.27). This concludes the proof of (4.31).

4.3. Sensitivity of $J$ in the presence of shocks. In this section we study the sensitivity of the functional $J$. This requires to study the sensitivity of the solutions $u$ of the conservation law with respect to variations associated with the generalized tangent vectors defined in the previous section.

Theorem 4.4. Assume that $f \in C^{2}(\mathbb{R})$ is a nonlinearity for which the weak entropy solution $u$ of (1.1) satisfies the hypothesis $(H)$. Then, for any $\delta f \in C^{2}(\mathbb{R})$, the following holds:

$$
\begin{aligned}
\delta J= & \lim _{\varepsilon \rightarrow 0^{+}} \frac{J(f+\varepsilon \delta f)-J(f)}{\varepsilon} \\
= & -T \int_{\{x<\varphi(T)\} \cup\{x>\varphi(T)\}} \partial_{x}(\delta f(u))(x, T)\left(u(x, T)-u^{d}(x)\right) d x \\
& -T \frac{\eta}{[u(\cdot, T)]_{\varphi(T)}}[\delta f(u(\cdot, T))]_{\varphi(T)},
\end{aligned}
$$

where

$$
\eta=\frac{1}{2}\left[\left(u(\cdot, T)-u^{d}(\varphi(T))\right)^{2}\right]_{\varphi(T)},
$$


if $u^{d}$ is continuous at $x=\varphi(T)$, and

$$
\eta= \begin{cases}\frac{1}{2}\left[\left(u(\cdot, T)-u^{d}\left(\varphi(T)^{+}\right)\right)^{2}\right]_{\varphi(T)}, & \text { if } \delta \varphi(T)>0, \\ \frac{1}{2}\left[\left(u(\cdot, T)-u^{d}\left(\varphi(T)^{-}\right)\right)^{2}\right]_{\varphi(T)}, & \text { if } \delta \varphi(T)<0,\end{cases}
$$

if $u^{d}$ has a jump discontinuity at $x=\varphi(T)$.

Remark 4.9. Note that, when $u^{d}$ is discontinuous at $x=\varphi(T)$, the value of $\eta$ in (4.45) depends on the sign of $\delta \varphi(T)$. This means that the expression of $\delta J$ in (4.43) is not necessarily the same if we take the limit as $\varepsilon \rightarrow 0$ for negative values of $\varepsilon$. The functional $J$ in this case is not Gateaux differentiable, in general.

Formula (4.43) coincides with formula (4.3) in the absence of shocks or when $[\delta f(u(\cdot, T))]_{\varphi(T)}=0$. In this case the Gateaux derivative of $J$ exists and it is as if no shocks were present. We prove later that, in fact, if this condition is satisfied, then the variation $\delta f$, to first order, does not move the shock position.

We briefly comment on the above result before giving the proof. Formula (4.43) provides easily a descent direction for $J$ when looking for a flux $f$ in the finite dimensional subspace $\mathcal{U}_{a d}^{M}$, defined in (2.6). In this case the derivative of the functional $J$ at $\alpha$ in the tangent direction $\delta \alpha=\left(\delta \alpha_{1}, \ldots, \delta \alpha_{M}\right) \in \mathbb{R}^{M}$ is given by

$$
\begin{aligned}
\delta J(\alpha)(\delta \alpha)= & -\sum_{m=1}^{M} \delta \alpha_{m}\left(T \int_{\mathbb{R}} \partial_{x}\left(f_{m}(u)\right)(x, T)\left(u(x, T)-u^{d}(x)\right) d x\right. \\
& \left.-\frac{\eta_{m}}{[u(\cdot, T)]_{\varphi(T)}} T\left[f_{m}(u(\cdot, T))\right]_{\varphi(T)}\right),
\end{aligned}
$$

where $\eta_{m}$ is the value of $\eta$ in (4.45) when $\delta f=f_{m}$.

Thus, the steepest descent direction for $J$ at $\alpha$ is given by

$$
\begin{aligned}
\delta \alpha_{m}= & T \int_{\mathbb{R}} \partial_{x}\left(f_{m}(u)\right)(x, T)\left(u(x, T)-u^{d}(x)\right) d x \\
& -\frac{\eta_{m}}{[u(\cdot, T)]_{\varphi(T)}} T\left[f_{m}(u(\cdot, T))\right]_{\varphi(T)}, \quad \text { for all } m \in 1, \ldots, M .
\end{aligned}
$$

Note, however, that this expression is not very useful in practice since it does not avoid computing the linearized system (4.27) for each $f_{m}$ with $m=1, \ldots, M$, in order to compute $\eta_{m}$, since the knowledge of the sign of $\delta \varphi(T)$ is required. This is due to the lack of Gateaux differentiability of $J$ that does not allow us to apply a proper adjoint methodology.

We finish this section with the proof of Theorem 4.4 .

Proof of Theorem 4.4. We first prove the following:

$$
\begin{aligned}
\delta J & =\lim _{\varepsilon \rightarrow 0^{+}} \frac{J(f+\varepsilon \delta f)-J(f)}{\varepsilon} \\
& =\int_{\{x<\varphi(T)\} \cup\{x>\varphi(T)\}}\left(u(x, T)-u^{d}(x)\right) \delta u(x, T) d x-\eta \delta \varphi(T),
\end{aligned}
$$

where the pair $(\delta u, \delta \varphi)$ is a generalized tangent vector of $(u(\cdot, T), \varphi(T))$ which solves the linearized problem (4.27) and $\eta$ is defined in (4.44)-(4.45).

Let us obtain (4.48) in the particular case where $u^{d}$ is discontinuous at $x=\varphi(T)$ and $\delta \varphi(T)>0$, the other cases being similar. Let $f_{\varepsilon}=f+\varepsilon \delta f$ and $\left(u_{\varepsilon}, \varphi_{\varepsilon}\right)$ be the solution of (4.25) associated to the flux $f_{\varepsilon}$. From Theorem 4.2, $\left(u_{\varepsilon}, \varphi_{\varepsilon}\right)$ is a 
path for a generalized tangent vector $(\delta u, \delta \varphi)$ satisfying (4.27). Thus, $\left(u_{\varepsilon}, \varphi_{\varepsilon}\right)$ is equivalent to a path of the form (4.26) and we have

$$
\begin{aligned}
\frac{1}{\varepsilon}\left(J\left(f_{\varepsilon}\right)-J(f)\right)= & \frac{1}{2 \varepsilon}\left(\int_{\mathbb{R}}\left(u_{\varepsilon}(x, T)-u^{d}(x)\right)^{2} d x-\int_{\mathbb{R}}\left(u(x, T)-u^{d}(x)\right)^{2} d x\right) \\
= & \int_{\{x<\varphi(T)\} \cup\{x>\varphi(T)+\varepsilon \delta \varphi(T)\}}\left(u(x, T)-u^{d}(x)\right) \delta u(x, T) d x \\
& +\frac{1}{2 \varepsilon} \int_{\varphi(T)}^{\varphi(T)+\varepsilon \delta \varphi(T)}\left(u(x, T)-[u]_{\varphi(T)}-u^{d}(x)\right)^{2} d x \\
& -\frac{1}{2 \varepsilon} \int_{\varphi(T)}^{\varphi(T)+\varepsilon \delta \varphi(T)}\left(u(x, T)-u^{d}(x)\right)^{2} d x+O(\varepsilon) \\
= & \int_{\{x<\varphi(T)\} \cup\{x>\varphi(T)+\varepsilon \delta \varphi(T)\}}\left(u(x, T)-u^{d}(x)\right) \delta u(x, T) d x \\
& +\frac{\delta \varphi(T)}{2}\left(u\left(\varphi(T)^{-}, T\right)-u^{d}\left(\varphi(T)^{+}\right)\right)^{2} \\
& -\frac{\delta \varphi(T)}{2}\left(u\left(\varphi(T)^{+}, T\right)-u^{d}\left(\varphi(T)^{+}\right)\right)^{2}+O(\varepsilon) \\
= & \int_{\{x<\varphi(T)\} \cup\{x>\varphi(T)+\varepsilon \delta \varphi(T)\}}\left(u(x, T)-u^{d}(x)\right) \delta u(x, T) d x \\
& -\delta \varphi(T)\left[\frac{1}{2}\left(u(x, T)-u^{d}\left(\varphi(T)^{+}\right)\right)^{2}\right]_{\varphi(T)}+O(\varepsilon) .
\end{aligned}
$$

We obtain (4.48) by passing to the limit as $\varepsilon \rightarrow 0$.

The final formula (4.43) is then a consequence of (4.48) and Theorem 4.3.

\section{Alternating Descent directions}

In this section we introduce a suitable decomposition of the vector space of tangent vectors associated to a flux function $f$. As we will see this decomposition allows us to obtain simplified formulas for the derivative of $J$ in particular situations. For example, we are interested in those variations $\delta f$ for which, at first order, the shock does not move at $t=T$.

Theorem 5.1. Assume that $f$ is a nonlinearity for which the weak entropy solution $u$ of (1.1) satisfies the hypothesis (H) and, in particular, the Rankine-Hugoniot condition (4.24). Let $\delta f \in C^{2}(\mathbb{R})$ be a variation of the nonlinearity $f$ and $(\delta u, \delta \varphi)$ the corresponding solution of the linearized system (4.27). Then, $\delta \varphi(T)=0$ if and only if

$$
[\delta f(u(\cdot, T))]_{\varphi(T)}=0 .
$$

Moreover, if condition (5.1) holds, the generalized Gateaux derivative of $J$ at $f$ in the direction $\delta f$ can be written as

$$
\delta J=-T \int_{\mathbb{R}} \partial_{x}(\delta f(u))(x, T)\left(u(x, T)-u^{d}(x)\right) d x .
$$

Proof. The characterization (5.1) follows from the identity (4.29). On the other hand, formula (5.2) is a particular case of identity (4.43). 
Remark 5.1. Formula (5.2) provides a simplified expression for the generalized Gateaux derivative of $J$ when considering variations $\delta f$ that do not move the shock position at $t=T$, at first order, i.e., those for which $\delta \varphi(T)=0$. These directions are characterized by formula (5.1).

In practice we assume that the fluxes $f$ are taken in the finite dimensional space $\mathcal{U}_{a d}^{M}$, defined in (2.6). The set $\mathcal{U}_{a d}^{M}$ can be parametrized by $\alpha=\left(\alpha_{1}, \ldots, \alpha_{M}\right) \in \mathbb{R}^{M}$. The condition (5.1) reads

$$
\sum_{m=1}^{M} \alpha_{m}\left[\delta f_{m}(u(\cdot, T))\right]_{\varphi(T)}=0,
$$

which defines a hyperplane in $\mathbb{R}^{M}$, corresponding to the set $\left(\alpha_{1}, \ldots, \alpha_{M}\right) \in \mathbb{R}^{M}$ orthogonal to

$$
\left(\left[\delta f_{1}(u(\cdot, T))\right]_{\varphi(T)}, \ldots,\left[\delta f_{M}(u(\cdot, T))\right]_{\varphi(T)}\right) \in \mathbb{R}^{M} .
$$

The results in Theorem [5.1 suggest the following decomposition of the tangent space $T_{\alpha}=\mathbb{R}^{M}$ constituted by the variations $\delta \alpha \in \mathbb{R}^{M}$ of a vector $\alpha \in \mathbb{R}^{M}$ :

$$
T_{\alpha}=T_{\alpha}^{1} \oplus T_{\alpha}^{2},
$$

where $T_{\alpha}^{1}$ is the subset constituted by the vectors $\left(\delta \alpha_{1}, \ldots, \delta \alpha_{M}\right)$ satisfying (5.3); and the one-dimensional subspace $T_{\alpha}^{2}$, generated by (5.4).

According to (4.46) and (5.2), the derivative of the functional $J$ at $\alpha$ in the tangent direction $\delta \alpha=\left(\delta \alpha_{1}, \ldots, \delta \alpha_{M}\right) \in T_{\alpha}^{1}$ is as in (4.4) and the steepest descent direction for $J$ at $\alpha$, when restricted to $T_{\alpha}^{1}$, is then given by (4.5).

Roughly speaking, we have obtained a steepest descent direction for $J$ among the variations which do not move the shock position at $t=T$, i.e., $\delta \varphi(T)=0$.

On the other hand, $T_{\alpha}^{2}$ defines a second class of variations which allows us to move the discontinuity of $u(x, T)$.

Note that, contrarily to [11] we do not consider the class of variations that, moving the shock, do not deform the solution away from it since this class is generically empty on the present context in which the nonlinearity is perturbed by a finite number of parameters.

We now define a strategy to obtain descent directions for $J$ at $f$. To illustrate this we consider the simplest case, i.e.,

$$
u^{d} \text { are Lipschitz continuous with a discontinuity at } x=x^{d} .
$$

As the solution $u$ has a shock discontinuity at the final time $t=T$, located at some point $x=\varphi(T)$, there are two possibilities, depending on the value of $\varphi(T)$ :

1. $\varphi(T) \neq x^{d}$. Then, we perturb $f$ with variations in $T_{\alpha}^{2}$ until we have $x^{d}=$ $\varphi(T)$. This is, in fact, a one-dimensional optimization problem that we can solve easily.

2. We already have $x^{d}=\varphi(T)$ and then we consider descent directions $\delta f \in$ $T_{f}^{1}$. To first order, these directions will not move the value of $\varphi$ at $t=T$, but will allow us to better fit the value of the solution to both sides of the shock.

In practice, the deformations of the second step will slightly move the position of the shock because the condition $\delta \varphi(T)=0$ that characterizes variations in the subspace $T_{f}^{1}$, only affects the position of the discontinuity at first order. Thus, one 
has to iterate this procedure to assure a simultaneous better placement of the shock and a better fitting of the value of the solution away from it.

This alternating descent method can be interpreted as a particular case of the relaxation method in which local minima are attained by optimizing in the directions given by the partial derivatives in a cyclic way. In our case, we divide the gradient in two components and optimize using them alternatively. Convergence of such algorithms is obtained under regularity assumptions on the functional and certain coerciveness conditions (see, for example [18]). In the present situation the functional is not even differentiable due to (4.43)- 4.45) and the convergence of this alternating method constitutes an interesting open problem.

In the next section we explain how to implement a descent algorithm following these ideas that, of course, can also be used in the case where the number of shocks of $u^{0}$ and $u^{d}$ is not necessarily one, or the same.

\section{Numerical approximation of the Descent Directions}

We have computed the gradient of the continuous functional $J$ in several cases ( $u$ smooth and having shock discontinuities) but, in practice, one has to look for descent directions for the discrete functional $J_{\Delta}$. In this section we discuss the various possibilities for searching them depending on the approach chosen (continuous versus discrete) and the degree of sophistication adopted.

We consider the following possibilities:

- The discrete approach: differentiable schemes.

- The discrete approach: non-differentiable schemes.

- The continuous approach.

- The continuous alternating descent method.

The last one is the new method we propose in this article by adapting the one introduced in [11] in the context of inverse design in which the control is the initial datum.

In the following section we present some numerical experiments that allow us to easily compare the efficiency of each method. As we shall see, the alternating descent method we propose, alternating the variations of the flux to sometimes move the shock and some others to correct the profile to both sides of it, is superior in several ways. It avoids the drawbacks of the other methods related either to the inefficiency of the differentiable methods to capture shocks or the difficulty of dealing with nondifferentiable schemes and the uncertainty of using "pseudolinearizations". As a consequence of this, the method we propose is much more robust and makes the functional $J$ decrease in a much more efficient way in a significantly smaller number of iterations.

The rest of this section is divided as follows: We first compute the gradient of the discrete cost functional when the numerical scheme chosen to approximate the nonlinear conservation law is differentiable. When the numerical scheme is not differentiable the gradient of the cost functional is not well defined and a descent direction must be computed in a different way. The usual approach is to consider a particular choice of the subgradient. We refer to [11] where this is treated in detail for the particular case of the Burgers equation. The last two subsections contain methods based on the continuous approach. More precisely, the third one describes the a priori more natural method based on the discretization of the continuous gradient while the fourth subsection is devoted to the new method introduced in 
this work in which we consider a suitable decomposition of the generalized tangent vectors.

We do not address here the convergence of descent algorithms when using the approximations of the gradients described above. In the present case, and taking into account that when dealing with the discrete functional $J_{\Delta}$ the number of control parameters is finite, one could prove convergence by using LaSalle's invariance principle and the cost functional as a Lyapunov functional, at least in the case of the discrete approach.

6.1. The discrete approach: Differentiable numerical schemes. Computing the gradient of the discrete functional $J_{\Delta}$ requires calculating one derivative of $J_{\Delta}$ with respect to each node of the mesh. This can be done in a cheaper way using the adjoint state. We illustrate it on the Lax-Friedrichs numerical scheme. Note that this scheme satisfies the hypothesis of Theorem 3.1 and therefore the numerical minimizers are good approximations of minimizers of the continuous problem. However, as the discrete functionals $J_{\Delta}$ are not necessarily convex the gradient methods could possibly provide sequences that do not converge to a global minimizer of $J_{\Delta}$. But this drawback and difficulty appears in most applications of descent methods in optimal design and control problems. As we will see, in the present context, the approximations obtained by gradient methods are satisfactory, although convergence is slow due to unnecessary oscillations that the descent method introduces.

Computing the gradient of $J_{\Delta}$, rigourously speaking, requires the numerical scheme (3.3) under consideration to be differentiable and, often, this is not the case. To be more precise, for the scalar equation (1.1) we can choose efficient methods which are differentiable (as the Lax-Friedrichs one) but this is not the situation for general systems of conservation laws in higher dimensions, as Euler equations. For such complex systems the efficient methods, such as Godunov, Roe, etc., are not differentiable (see, for example [22] or 28]) thus making the approach in this section useless.

We observe that when the 3-point conservative numerical approximation scheme (3.3) used to approximate the scalar equation (1.1) has a differentiable numerical flux function $g$, then the linearization is easy to compute. We obtain

$$
\left\{\begin{aligned}
& \delta u_{j}^{n+1}=\delta u_{j}^{n}-\lambda\left(\delta g_{j+1 / 2}^{n}+\partial_{1} g_{j+1 / 2}^{n} \delta u_{j}^{n}+\partial_{2} g_{j+1 / 2}^{n} \delta u_{j+1}^{n}\right. \\
&\left.-\delta g_{j-1 / 2}^{n}-\partial_{1} g_{j-1 / 2}^{n} \delta u_{j-1}^{n}-\partial_{2} g_{j-1 / 2}^{n} \delta u_{j}^{n}\right), \\
& j \in \mathbb{Z}, \quad n=0, \ldots, N .
\end{aligned}\right.
$$

Here, $\delta g_{j+1 / 2}^{n}=\delta g\left(u_{j}^{n}, u_{j+1}^{n}\right)$ where $\delta g$ represents the Gateaux derivative of the numerical flux $g(u, v)$ with respect to the flux $f$. Note that (6.1) is in fact a suitable discretization of (4.10).

On the other hand, for any variation $\delta f \in \mathcal{U}_{a d}$ of $f$, the Gateaux derivative of the cost functional defined in (3.2) is given by

$$
\delta J_{\Delta}=\Delta x \sum_{j \in \mathbb{Z}}\left(u_{j}^{N+1}-u_{j}^{d}\right) \delta u_{j}^{N+1},
$$

which is the discrete version of (4.11).

It is important to observe that here we cannot solve system (6.1) to obtain the discrete version of (4.12), as in the continuous case, since it is based on a characteristic construction which is difficult to translate at the discrete level. Thus, 
unlike in the continuous case, we must introduce a discrete adjoint system to obtain a simplified expression of the Gateaux derivative (6.2).

The discrete adjoint system for any differentiable flux function $g$ is,

$$
\left\{\begin{array}{l}
p_{j}^{n}=p_{j}^{n+1}+\lambda\left(\partial_{1} g_{j+1 / 2}^{n}\left(p_{j+1}^{n+1}-p_{j}^{n+1}\right)+\partial_{2} g_{j-1 / 2}^{n}\left(p_{j}^{n+1}-p_{j-1}^{n+1}\right)\right), \\
p_{j}^{N+1}=u_{j}^{N+1}-u_{j}^{d}, \quad j \in \mathbb{Z}, \quad n=0, \ldots, N .
\end{array}\right.
$$

In fact, when multiplying the equations in (6.1) by $p_{j}^{n+1}$ and adding in $j \in \mathbb{Z}$ and $n=0, \ldots, N$, the following identity is easily obtained:

$$
\Delta x \sum_{j \in \mathbb{Z}}\left(u_{j}^{N+1}-u_{j}^{d}\right) \delta u_{j}^{N+1}=-\Delta x \lambda \sum_{n=0}^{N} \sum_{j \in \mathbb{Z}}\left(\delta g_{j+1 / 2}^{n}-\delta g_{j-1 / 2}^{n}\right) p_{j}^{n+1} .
$$

This is the discrete version of the identity

$$
\int_{\mathbb{R}}\left(u(x, T)-u^{d}(x)\right) \delta u(x, T) d x=-\int_{0}^{T} \int_{\mathbb{R}} \partial_{x}\left(f^{\prime}(u(x, t))\right) p(x, t) d x d t,
$$

which holds in the continuous case, and it allows us to simplify the derivative of the discrete cost functional. Note also that (6.3) is also a particular discretization of the adjoint system

$$
\begin{cases}-\partial_{t} p-f^{\prime}(u) \partial_{x} p=0, & x \in \mathbb{R}, t \in(0, T) \\ p(x, T)=u(x, T)-u^{d}(x), & x \in \mathbb{R}\end{cases}
$$

In view of (6.3) and (6.4), for any variation $\delta f \in \mathcal{U}_{a d}$ of $f$, the Gateaux derivative of the cost functional defined in (3.2) is given by

$$
\delta J_{\Delta}=\Delta x \sum_{j \in \mathbb{Z}}\left(u_{j}^{N+1}-u_{j}^{d}\right) \delta u_{j}^{N+1}=-\Delta x \lambda \sum_{n=0}^{N} \sum_{j \in \mathbb{Z}}\left(\delta g_{j+1 / 2}^{n}-\delta g_{j-1 / 2}^{n}\right) p_{j}^{n+1},
$$

which is the discrete version of

$$
\delta J=-\int_{0}^{T} \int_{\mathbb{R}} \partial_{x}(\delta f(u(x, t))) p(x, t) d x d t .
$$

Formula (6.5) allows us to obtain easily the steepest descent direction for $J_{\Delta}$. In fact, given $f \in \mathcal{U}_{a d}^{M}$ with $f=\sum_{m=1}^{M} \alpha_{m} f_{m}$ for some coefficients $\alpha_{m} \in \mathbb{R}$, and

$$
\delta f=\sum_{m=1}^{M} \delta \alpha_{m} f_{m}
$$

making explicit the dependence of the numerical flux $g$ on $f$ by writing $g(u, v)=$ $g(u, v ; f)$, we have

$$
\delta g_{j+1 / 2}^{n}=\delta g\left(u_{j}^{n}, u_{j+1}^{n} ; f\right)=\partial_{f} g\left(u_{j}^{n}, u_{j+1}^{n} ; f\right) \delta f=\sum_{m=1}^{M} \partial_{f} g\left(u_{j}^{n}, u_{j+1}^{n} ; f\right) f_{m} \delta \alpha_{m} .
$$

When substituting in (6.5), we obtain

$$
\delta J_{\Delta}=-\Delta x \lambda \sum_{n=0}^{N} \sum_{j \in \mathbb{Z}} \sum_{m=1}^{M}\left(\partial_{f} g\left(u_{j}^{n}, u_{j+1}^{n} ; f\right) f_{m}-\partial_{f} g\left(u_{j-1}^{n}, u_{j}^{n} ; f\right) f_{m}\right) \delta \alpha_{m} p_{j}^{n+1}
$$


and a descent direction for $J^{\Delta}$ at $f_{\Delta}$ is given by

$$
\delta \alpha_{m}=\Delta x \lambda \sum_{n=0}^{N} \sum_{j \in \mathbb{Z}}\left(\partial_{f} g\left(u_{j}^{n}, u_{j+1}^{n} ; f\right) f_{m}-\partial_{f} g\left(u_{j-1}^{n}, u_{j}^{n} ; f\right) f_{m}\right) p_{j}^{n+1} .
$$

Remark 6.1. At this point it is interesting to compare formulas (6.6) and (6.7) with their corresponding expressions at the continuous level, i.e., formulas (4.4) and (4.5), respectively. The discrete formulas are obtained by projecting formula (6.5) into the finite dimensional subspace $\mathcal{U}_{a d}^{M}$, while the continuous ones come from the projection of the simplified expression (4.4). As we have said before, we do not know how to obtain a discrete version of (4.4) from (6.5).

As a consequence, the discrete formula (6.7) involves more computations than expected for any discrete version of (4.5), and it even requires solving a discrete adjoint system. From the computational point of view, this makes a priori preferable to use as a descent direction a suitable discretization of (4.5) rather than (6.7).

Remark 6.2. We do not address here the problem of the convergence of these adjoint states towards the solutions of the continuous adjoint system. Of course, this is an easy matter when $u$ is smooth but is far from being trivial when $u$ has shock discontinuities. Whether or not these discrete adjoint systems, as $\Delta \rightarrow 0$, allow reconstructing the complete adjoint system, with the inner Dirichlet condition along the shock, constitutes an interesting problem for future research. We refer to [23] for preliminary work on this direction.

6.2. The continuous approach. This method is based on the result stated in Theorem 4.4 indicating that the sensitivity of the functional is obtained by formula (4.3). In particular, when considering the finite dimensional subspace $\mathcal{U}_{a d}^{M}$, the steepest descent direction is given by (4.4). A tentative descent direction for the discrete functional is then obtained by a numerical approximation of (4.4). One possible choice is to take, for each $m \in 1, \ldots, M$,

$$
\delta \alpha_{m}=T \sum_{j \in \mathbb{Z}}\left(f_{m}\left(u_{j+1}^{N+1}\right)-f_{m}\left(u_{j}^{N+1}\right)\right)\left(\frac{u_{j}^{N+1}+u_{j+1}^{N+1}}{2}-\frac{u_{j}^{d}+u_{j+1}^{d}}{2}\right),
$$

where $u_{j}^{n}$ is obtained from a suitable conservative numerical scheme regardless of its differentiability. This formula is obviously consistent with the components of the steepest descent direction in (4.5), if no shocks are present.

6.3. The alternating descent method. Here we propose a new method inspired by the results in Theorem 5.1 and the discussion thereafter. We shall refer to this new method as the alternating descent method, that was first introduced in [1] in the context of an optimal control problem for the Burgers equation where the control variable is the initial datum.

To fix ideas we assume that we look for $f$ in the finite dimensional subspace $\mathcal{U}_{a d}^{M}$. We also assume that both the target $u^{d}$ and the initial datum $u^{0}$ are Lipschitz continuous functions having one single shock discontinuity. But these ideas can be applied in a much more general context in which the number of shocks is larger and do not even necessarily coincide.

To initiate the optimization iterative process we choose a nonlinearity $f$ in such a way that the solution at time $t=T$ has a profile similar to $u^{d}$, i.e., it is a Lipschitz continuous function with a single discontinuity of negative jump, located on an 
arbitrary point $x \in \mathbb{R}$. The main idea now is to build a minimizing sequence of $J$ alternating the following two steps: First we perturb the flux $f$ so to move the discontinuity of the solution $u$ of (1.1) at time $t=T$, regardless of its value to both sides of the discontinuity. Once this is done we perturb the resulting $f$ so that the position of the discontinuity is kept fixed and alter the value of $u(x, T)$ to both sides of it. This is done by decomposing the set of variations associated to $f$ into the two subsets introduced in (5.5), considering alternatively variations $\delta_{\alpha} \in T_{\alpha}^{1}$ and $\delta_{\alpha} \in T_{\alpha}^{2}$ as descent directions.

For a given initialization of $f$, in each step of the descent iteration process we proceed in the following three sub-steps:

(1) Determine the subspaces $T_{\alpha}^{1}$ and $T_{\alpha}^{2}$ by computing the vector (5.4). Note that $T_{\alpha}^{2}$ is in fact a one-dimensional subspace.

(2) We consider the vector (5.4) that generates $T_{\alpha}^{2}$, and choose the optimal step, so to minimize the functional in that direction of variation of the nonlinearity $f$. This involves a one-dimensional optimization problem that we can solve with a classical method (bisection, Armijo's rule, etc.). In this way we obtain the best location of the discontinuity on that step.

(3) We then use the descent direction $\delta \alpha \in T_{\alpha}^{1}$ to modify the value of the solution at time $t=T$ to both sides of the discontinuity. Here, we can again estimate the step size by solving a one-dimensional optimization problem or simply take a constant step size.

\section{Numerical EXPERIMENTS}

In this section we present some numerical experiments which illustrate the results obtained in an optimization model problem with each one of the numerical methods described in this paper.

The following numerical methods are considered:

(1) The discrete approach with the Lax-Friedrichs scheme. The optimization procedure is based on the steepest descent method and the descent directions are computed using the adjoint approach.

(2) The discrete approach with the Roe scheme. It has the numerical flux,

$$
g^{R}(u, v)=\frac{1}{2}(f(u)+f(v)-|A(u, v)|(v-u)),
$$

where the matrix $A(u, v)$ is a Roe linearization which is an approximation of $f^{\prime}$. In the scalar case under consideration $A(u, v)$ is a function given by,

$$
A(u, v)=\left\{\begin{array}{l}
\frac{f(u)-f(v)}{u-v}, \text { if } u \neq v \\
f^{\prime}(u), \text { if } u=v
\end{array}\right.
$$

The optimization procedure is again based on the steepest descent method and the descent directions are computed using the adjoint approach. We recall that unlike the Lax-Friedrichs scheme this one is not differentiable and the adjoint system is obtained formally (see [19, 11]).

(3) The continuous approach with the Roe scheme. We use the method described in subsection 6.2. In this case, as we discretize the continuous adjoint system, the use of a differentiable or a nondifferentiable scheme is not relevant to approximate the direct problem. However, in practice, a numerical scheme based on a pseudo-linearization of the scheme used for the direct problem is usually more efficient. This pseudo-linearization is 
usually obtained by considering a particular choice of the subdifferential in the regions where the scheme is not differentiable.

(4) The continuous approach with the Roe scheme using the generalized tangent vectors decomposition and the alternating descent method described in subsection 6.3 .

We have chosen as computational domain the interval $(-4,4)$ and we have taken as boundary condition in (1.1), at each time step $t=t^{n}$, the value of the initial data at the boundary. This can be justified if we assume that both the initial and final data $u^{0}, u^{d}$ take the same constant value $\bar{u}$ in a sufficiently large neighborhood of the boundary $x= \pm 4$, and the value of $f^{\prime}(\bar{u})$ does not become very large in the optimization process, due to the finite speed of propagation. A similar procedure is applied for the adjoint equation.

In our experiments we assume that the flux $f$ is a polynomial. The relevant part of the flux function is its derivative since it determines the slope of the characteristic lines. Thus, we take $f^{\prime}$ of the form

$$
f^{\prime}(u)=\alpha_{1} P_{1}(u)+\alpha_{2} P_{2}(u)+\cdots+\alpha_{6} P_{6}(u),
$$

where $\alpha_{j}$ are some real coefficients and $P_{j}(u)$ are the Legendre polynomials, orthonormal with respect to the $L^{2}(a, b)$-norm. The interval $[a, b]$ is chosen in such a way that it contains the range of $u_{0}$, and therefore the range of the solutions $u$ under consideration. To be more precise, we take $[a, b]=[0,1]$ and then

$$
\begin{aligned}
& P_{1}(u)=1 \\
& P_{2}(u)=\sqrt{12}(u-1 / 2), \\
& P_{3}(u)=\sqrt{80}\left(3 / 2 u^{2}-3 / 2 u+1 / 4\right), \\
& P_{4}(u)=\sqrt{448}\left(5 / 2 u^{3}-15 / 4 u^{2}+3 / 2 u-1 / 8\right) \\
& P_{5}(u)=\sqrt{2304}\left(35 / 8 u^{4}-35 / 4 u^{3}+45 / 8 u^{2}-5 / 4 u+1 / 16\right), \\
& P_{6}(u)=\sqrt{11264}\left(63 / 8 u^{5}-315 / 16 u^{4}+35 / 2 u^{3}-105 / 16 u^{2}+15 / 16 u-1 / 32\right) .
\end{aligned}
$$

Experiment 1. We first consider a piecewise constant initial datum $u^{0}$ and target profile $u^{d}$ given by

$$
\begin{aligned}
& u^{0}=\left\{\begin{array}{l}
1 \text { if } x<1, \\
0 \text { if } x \geq 1,
\end{array}\right. \\
& u^{d}=\left\{\begin{array}{l}
1 \text { if } x<0, \\
0 \text { if } x \geq 0,
\end{array}\right.
\end{aligned}
$$

and the time $T=1$.

We solve the optimization problem (3.9) with the above described different methods. The algorithms are initialized either with $f=0$ or the nonlinearity corresponding to the Burgers equation,

$$
f(u)=u^{2} / 2 .
$$

Note that the solution $u$ of the corresponding scalar conservation laws can be computed explicitly for this choice of the initial datum and the nonlinearities. If $f=0$, $u(x, t)=u^{0}(x)$ while for the Burgers equation $u(x, 1)=1$ if $x \leq 3 / 2$ and zero otherwise. This function $u(x, 1)$ has the same profile as $u^{d}$ but the discontinuity is shifted. Thus the optimization method should find a nonlinearity that simply moves the discontinuity of $u(x, t)$ to the same place as the one of $u^{d}(x)$ at time $t=1$. 
Note also that any nonlinearity for which $f^{\prime}(0)<f^{\prime}(1)$ and $f(0)-f(1)=1$ is a minimizer for $J$ whose value is zero. Indeed, in that case $u$ is a piecewise shock solution that, at the final time, is located precisely at the point $x=0$. In particular, an obvious minimizer is obtained by the linear flux $f(u)=-u$.

Recall that the stability of the numerical scheme chosen to approximate the conservation law depends on the CFL condition, which relates $\Delta t / \Delta x$ with the speed of propagation, characterized by $f^{\prime}(u)$. Despite this, the discrete optimization problem still has a sense without that constraint, but the convergence of the discrete optima is not guaranteed in this case.

We have first conducted the experiment without any a priori bound on the size of the admissible nonlinearities. In our experiment, regardless of the method employed (Lax-Friedrichs, Roe, continuous, etc.), the nonlinearity obtained after the optimization process turns out to be very sensitive to the CFL condition. This effect is more evident for the Lax-Friedrichs scheme. In Figure 3 we show the derivative of the nonlinearity $f^{\prime}$ obtained with $\Delta t / \Delta x=1 / 2,1 / 4,1 / 8$. We observe that its $L^{\infty}$-norm increases as $\Delta t / \Delta x$ decreases. Thus, roughly speaking, the CFL condition acts as an active constraint and the method tends to saturate the admissible $W^{1, \infty}$-bound guaranteeing stability. This also indicates the necessity of including a bound in the $W^{1, \infty}$-norm of the nonlinearities $f$ in order to ensure the convergence of the method, as required in our theoretical results.

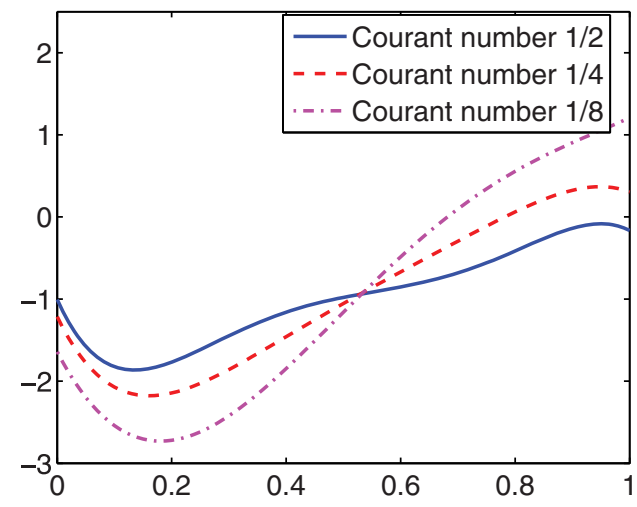

Figure 3. Experiment $1 . f^{\prime}(s)$ obtained after 30 iterations of the gradient method, for the unpenalized functional (3.2), with the Lax-Friedrichs scheme and for different values of the Courant number $\Delta x / \Delta t=1 / 2,1 / 4,1 / 8$. The algorithm is initialized with $f=0$.

In order to avoid this unstability, instead of considering a constrained optimization problem including a bound on $f$, we incorporate a penalization term in the functional, as indicated in (2.5). The modified functional penalizes $f^{\prime}$ in the $L^{2}(0,1)$-norm. Note that the chosen norm is not a very important issue at this level since we are looking for nonlinearities in a finite dimensional set.

Thus, we effectively minimize the functional

$$
J(u)=\int_{\mathbb{R}}\left|u(x, T)-u^{d}(x)\right|^{2} d x+\frac{1}{10} \int_{0}^{1}\left|f^{\prime}(s)\right|^{2} d s .
$$


Of course, the nonlinearity $f(u)=-u$ is no longer a minimizer of this functional but minimizers are likely to be close.

In Table 1 we present the nonlinearities $f$ obtained with the different methods and the corresponding values of $f(0)-f(1)$.

TABle 1. Experiment 1. Values for the parameters found after 12 iterations of the descent algorithm with the different methods. The last column contains the value $f(0)-f(1)$, which must be 1 for the minimizers of the continuous functional without penalization. We assume that the Courant number is $\Delta t / \Delta x=0.5$ and the algorithm is initialized with $f_{i n i}=0$ in the first two tables and $f_{i n i}=u^{2} / 2$ in the last one.

\begin{tabular}{l||c|c|c|c|c|c||c|}
$\Delta x=\frac{1}{20}$ & $\alpha_{1}$ & $\alpha_{2}$ & $\alpha_{3}$ & $\alpha_{4}$ & $\alpha_{5}$ & $\alpha_{6}$ & $f(0)-f(1)$ \\
\hline \hline Lax-Fr. & -0.9082 & 0.2149 & 0.2014 & -0.1127 & 0.0675 & -0.0268 & 0.9082 \\
Roe & -0.9354 & 0.1347 & 0.1797 & -0.1048 & 0.0176 & 0.0059 & 0.9354 \\
Cont. & -0.9240 & 0.1575 & 0.2299 & -0.2108 & 0.0226 & -0.0139 & 0.9240 \\
Alt. & -0.9832 & 0.3000 & 0.0054 & -0.0046 & -0.0029 & 0.0078 & 0.9832 \\
\hline$\Delta x=\frac{1}{40}$ & $\alpha_{1}$ & $\alpha_{2}$ & $\alpha_{3}$ & $\alpha_{4}$ & $\alpha_{5}$ & $\alpha_{6}$ & $f(0)-f(1)$ \\
\hline \hline Lax-Fr. & -0.9176 & 0.0681 & 0.1997 & -0.1167 & 0.0656 & 0.0237 & 0.9176 \\
Roe & -0.9648 & 0.0171 & 0.0797 & -0.1415 & 0.0183 & 0.0480 & 0.9354 \\
Cont. & -0.9465 & 0.0234 & 0.1304 & -0.2533 & -0.0136 & 0.1058 & 0.9465 \\
Alt. & -0.9865 & 0.1227 & 0.0831 & -0.1129 & -0.0407 & 0.0404 & 0.9865 \\
\hline$\Delta x=\frac{1}{20}$ & $\alpha_{1}$ & $\alpha_{2}$ & $\alpha_{3}$ & $\alpha_{4}$ & $\alpha_{5}$ & $\alpha_{6}$ & $f(0)-f(1)$ \\
\hline \hline Lax-Fr. & -0.9136 & 0.2220 & 0.1907 & -0.1070 & 0.0666 & -0.0320 & 0.9136 \\
Roe & -0.9536 & 0.1403 & 0.1201 & -0.0611 & 0.0241 & -0.0318 & 0.9536 \\
Cont. & -0.9125 & 0.1879 & 0.3727 & -0.1332 & -0.0488 & -0.1111 & 0.9125 \\
Alt. & -0.9782 & 0.3017 & 0.0404 & -0.0288 & 0.0169 & -0.0267 & 0.9782 \\
\hline
\end{tabular}

In Figure 4 we show the value of the functional after the first 12 iterations for $\Delta x=1 / 20,1 / 40$. We see that the alternating method obtains lower values of the functional in fewer iterations. Note also that the situation is similar if we consider finer meshes or different initializations.

Experiment 2. Now we consider a piecewise constant initial datum $u^{0}$ and target profile $u^{d}$ given by

$$
\begin{gathered}
u^{0}=\left\{\begin{array}{l}
1 \text { if } x<-2, \\
0 \text { if } x \geq-2,
\end{array}\right. \\
u^{d}=\left\{\begin{array}{l}
1 \text { if } x<2, \\
0 \text { if } x \geq 2,
\end{array}\right.
\end{gathered}
$$

and the time $T=1$.

We solve the optimization problem (3.9) with the above described different methods. The algorithms are initialized with $f=0$.

The main difference with the previous experiment is that the discontinuity of the initial datum and the target are now far away, and shifted to the right, unlike the previous example. In fact, any nonlinearity $f$, with $f^{\prime}(0)<f^{\prime}(1)$, which satisfies $f(0)-f(1)=-4$ is a minimizer of the continuous functional $J$ without penalization. 

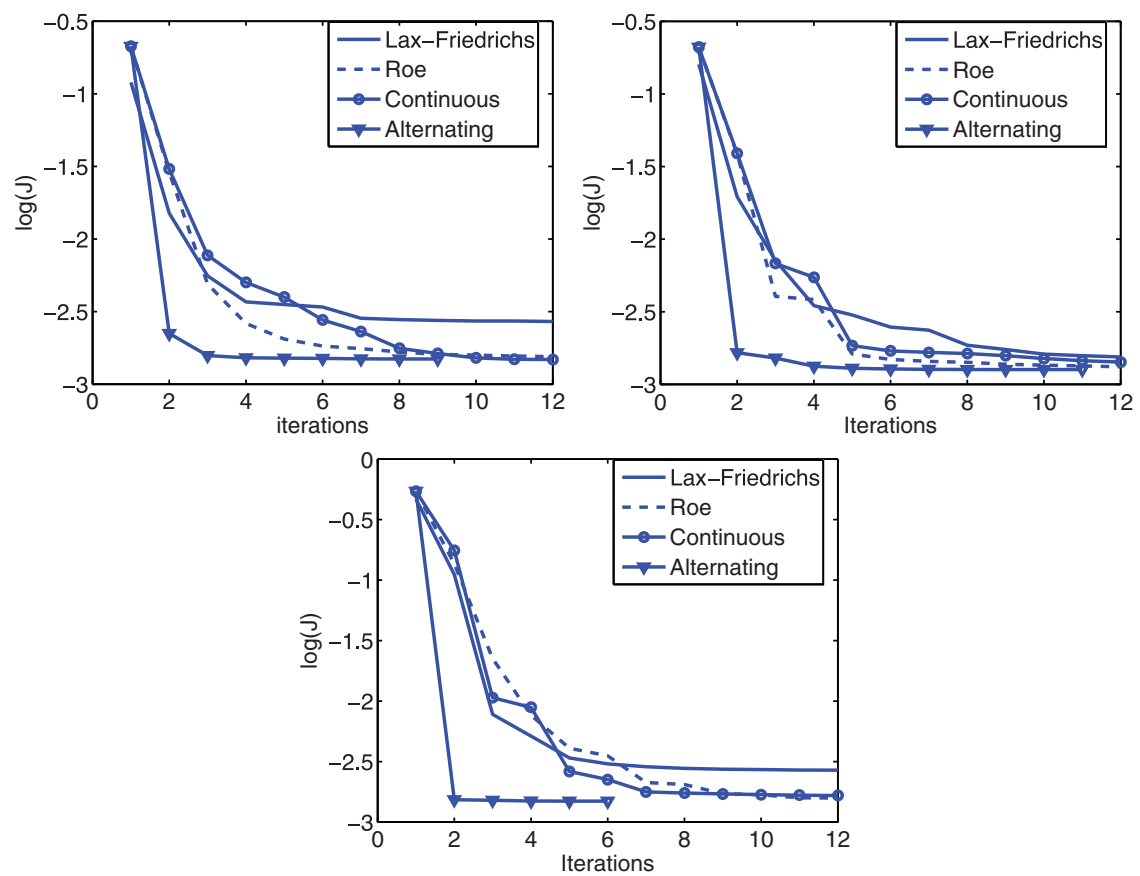

Figure 4. Experiment 1. Log of the functional versus the number of iterations for the different methods. $\Delta t / \Delta x=1 / 20$ (upper left) and $1 / 40$ (upper right) with initialization $f=0$. The lower figure corresponds to the initialization $f(u)=u^{2} / 2$ and $\Delta x=1 / 20$.

Another important remark is that the characteristic lines corresponding to the minimizers will have higher slopes and, therefore, we will require a smaller value of the Courant number to achieve stability. We take $\Delta t / \Delta x=0.1$.

In Figure 5 we show the value of the functional after the first 20 iterations for $\Delta x=1 / 20$.

TABLE 2. Experiment 2. Optimal values for the parameters with the different methods $(\Delta x=1 / 20)$.

\begin{tabular}{l||c|c|c|c|c|c||c|} 
Par. & $\alpha_{1}$ & $\alpha_{2}$ & $\alpha_{3}$ & $\alpha_{4}$ & $\alpha_{5}$ & $\alpha_{6}$ & $f(0)-f(1)$ \\
\hline \hline L-Fr. & 3.0128 & -0.7864 & -0.7380 & 0.0834 & 0.0728 & 0.0011 & -3.0128 \\
Roe & 3.0236 & -0.9298 & -0.6349 & 0.1732 & 0.0640 & -0.0505 & -3.0236 \\
Cont. & 2.9050 & -0.9717 & -0.8027 & 0.3290 & 0.0037 & -0.0700 & -2.9050 \\
Alt. & 3.2532 & -0.5759 & -0.5268 & 0.2900 & -0.0408 & -0.0963 & -3.2532 \\
\hline
\end{tabular}




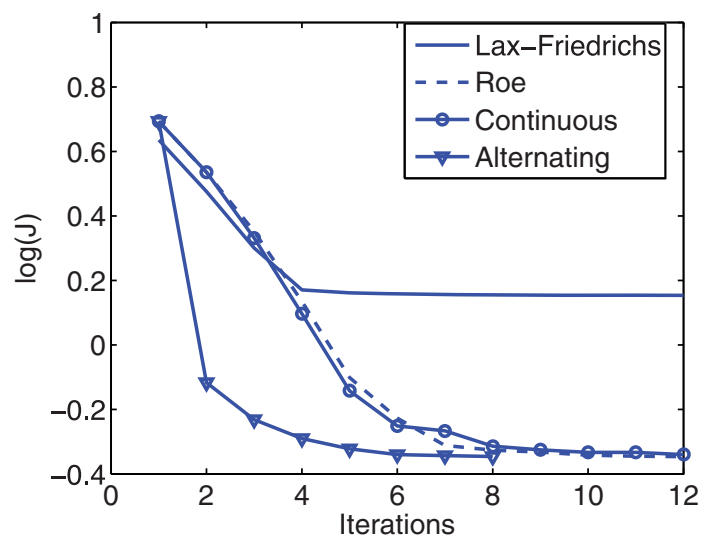

Figure 5. Experiment 2. Log of the functional versus the number of iterations for the different methods. We assume that the discretization parameter is $\Delta x=1 / 20$.

Experiment 3. Now we consider

$$
\begin{gathered}
u^{0}=\left\{\begin{array}{c}
\sin (2 \pi x) \text { if }|x|<2, \\
0 \text { if }|x| \geq 2,
\end{array}\right. \\
u^{d}=\left\{\begin{array}{l}
\sin (2 \pi(x-1 / 2)) \text { if }|x-1 / 2|<2, \\
0 \text { if }|x-1 / 2| \geq 2,
\end{array}\right.
\end{gathered}
$$

and the time $T=1$.

Note that the linear flux $f(u)=u / 2$ is a minimizer of the continuous functional. We solve the optimization problem (3.9) with the above described different methods. The algorithms are initialized with (7.4).

The main difference with the previous experiment is that there is no discontinuity in the initial datum nor in the target. However, the solution generates shocks due to the oscillations of $u^{0}$.

In Figure 6 we show the value of the functional after the first 20 iterations for $\Delta x=1 / 20$ and $\Delta t / \Delta x=0.5$.

TABle 3. Experiment 3. Optimal values for the parameters with the different methods

\begin{tabular}{l||c|c|c|c|c|c|} 
parameters & $\alpha_{1}$ & $\alpha_{2}$ & $\alpha_{3}$ & $\alpha_{4}$ & $\alpha_{5}$ & $\alpha_{6}$ \\
\hline \hline Lax-Friedrichs & 0.4601 & 0.5786 & 0.0016 & -0.0954 & -0.0034 & -0.0383 \\
Roe & -0.5497 & 1.4065 & -0.0901 & 0.1191 & -0.0165 & 0.0446 \\
Continuous & -0.5326 & 1.0487 & -0.0246 & 0.0244 & -0.0020 & 0.0030 \\
Alternating & 0.4902 & 0.1256 & 0.0276 & -0.0804 & 0.0037 & -0.0110 \\
\hline
\end{tabular}




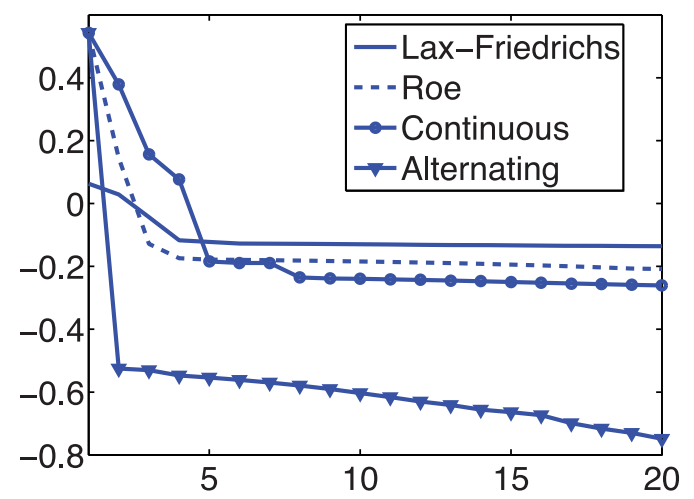

FiguRE 6. Experiment 3. Log of the functional versus the number of iterations for the different methods.
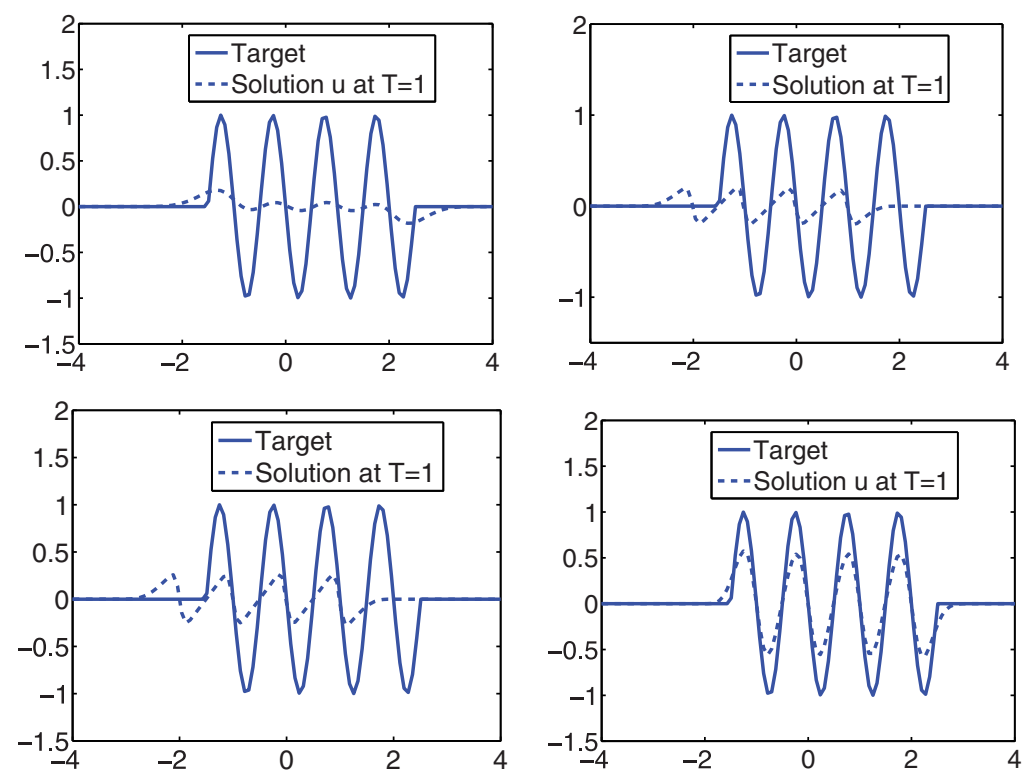

FiguRE 7. Experiment 3. Target and solution at time $T=1$ with the optimal $f$ found with the Lax-Friedrichs (upper left), Roe (upper right), continuous (lower left) and alternating (lower right) methods.

Experiment 4. Now we consider

$$
\begin{gathered}
u^{0}=\left\{\begin{array}{l}
\max \left(-(x-1)^{2} / 8+1 / 2,0\right) \text { if } x<1, \\
\min \left((x-1)^{2} / 8-1 / 2,0\right) \text { if } x \geq 1,
\end{array}\right. \\
u^{d}=\left\{\begin{array}{l}
\max \left(-(x+1 / 2)^{2} / 8+1 / 2,0\right) \text { if } x<-1 / 2, \\
\min \left((x+1 / 2)^{2} / 8-1 / 2,0\right) \text { if } x \geq-1 / 2,
\end{array}\right.
\end{gathered}
$$

and the time $T=1$. 


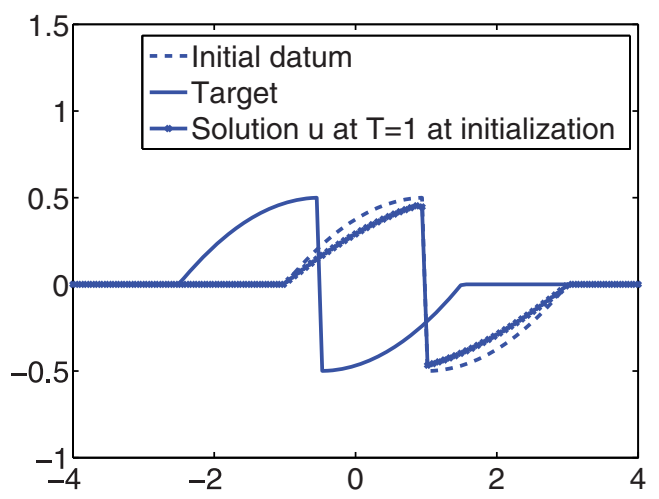

Figure 8. Experiment 4. Initial datum $u^{0}$, final datum $u^{d}$ and the solution $u(x, T)$ with the initialization parameters $\alpha_{j}$.

In this case the flux $f(u)=-3 / 2 u$ is a minimizer of the continuous functional without penalization. We solve the optimization problem (3.9) with the above described different methods. The algorithms are initialized with (7.4).

In Figure 9 we show the value of the functional after the first 20 iterations for $\Delta x=1 / 20$.

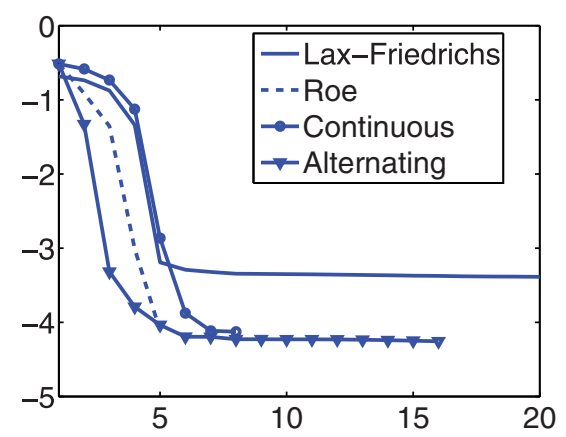

Figure 9. Experiment 4. Log of the functional versus the number of iterations for the different methods.

TABle 4. Experiment 4. Optimal values for the parameters with the different methods

\begin{tabular}{l||c|c|c|c|c|c|} 
parameters & $\alpha_{1}$ & $\alpha_{2}$ & $\alpha_{3}$ & $\alpha_{4}$ & $\alpha_{5}$ & $\alpha_{6}$ \\
\hline \hline Lax-Friedrichs & -1.4628 & 0.9797 & -0.0459 & 0.0006 & -0.0030 & 0 \\
Roe & -1.4591 & 1.1609 & -0.0695 & 0.0257 & -0.0072 & 0.0043 \\
Continuous & -1.4564 & 1.1437 & -0.0451 & 0.0196 & -0.0031 & 0.0028 \\
Alternating & -1.4518 & 0.9447 & -0.0927 & -0.0047 & -0.0108 & -0.0005 \\
\hline
\end{tabular}



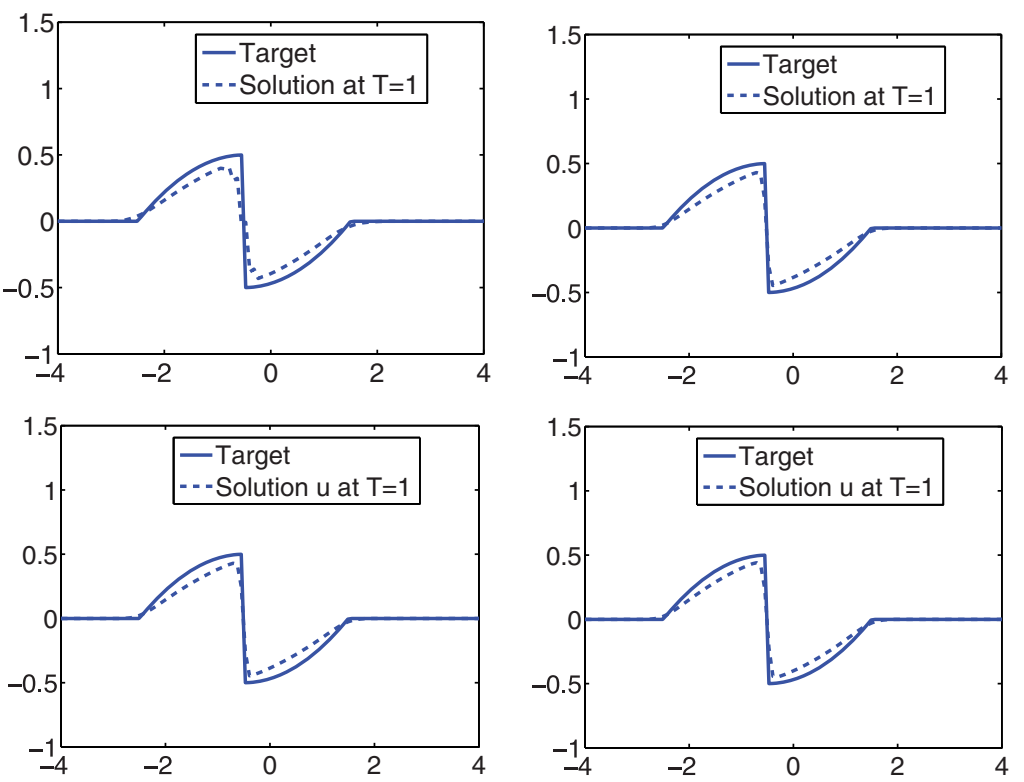

Figure 10. Experiment 4. Target and solution at time $T=1$ with the optimal $f$ found with the Lax-Friedrichs (upper left), Roe (upper right), continuous (lower left) and alternating (lower right) methods.

\section{Conclusions}

In this paper we have considered the numerical approximation of a flux optimization problem for a one-dimensional scalar conservation law. To compute the gradient of the cost functional we have discussed both the discrete approach and the continuous one for smooth solutions and solutions in presence of a single shock. The discrete approach requires solving an adjoint system while the continuous one does not. More precisely, when dealing with smooth solutions, we have deduced a new formula for the gradient of the continuous functional which does not require solving the associated adjoint system.

In the presence of a shock, the gradient calculus requires a suitable linearization of the solutions of the conservation laws, based on the generalized tangent vectors introduced in [9]. This provides a new formulation of the gradient which takes into account both small variations on the value of the solutions and small variations on the position of the discontinuity. Due to the different nature of the admissible variations it seems natural to consider separately descent directions producing changes on the shock position and those that do not move it. In this way, we have introduced a new optimization strategy where these directions are used alternatively in the optimization process.

From a numerical point of view, both approaches (the continuous and the discrete one) provide good results. However, the continuous approach requires less 
computations, due to the fact that the adjoint system is not solved. The alternating descent directions strategy yields better results than the other methods, when the displacement of the shocks is relevant, mainly in the first iterations.

In experiment 3 above we also see that, when using a descent direction based on a displacement of the shock, we can avoid some local minima in some specific situations.

\section{ApPENDiX}

In this final section we prove Theorem 4.2 The proof is divided into several steps. In step 1 we prove that, for all $t \in[0, T]$ the path $\left(u_{\varepsilon}(\cdot, t), \varphi_{\varepsilon}(t)\right)$ is a regular variation for $(u(\cdot, t), \varphi(t))$, in the sense of Definition 4.1. Once this is proved we have to see that its associated generalized tangent vector is $(\delta u, \delta \varphi)$, for all $t \in[0, T]$, i.e.,

$$
\lim _{\varepsilon \rightarrow 0^{+}} \frac{1}{\varepsilon}\left\|u_{\varepsilon}(\cdot, t)-u(\cdot, t)-\varepsilon \delta u(\cdot, t)-[u]_{\varphi(t)} \chi_{[\varphi(t), \varphi(t)+\varepsilon \delta \varphi(t)]}\right\|_{L^{1}(\mathbb{R})}=0,
$$

if $\delta \varphi>0$, and

$$
\lim _{\varepsilon \rightarrow 0^{+}} \frac{1}{\varepsilon}\left\|u_{\varepsilon}(\cdot, t)-u(\cdot, t)-\varepsilon \delta u(\cdot, t)-[u]_{\varphi(t)} \chi_{[\varphi(t)+\varepsilon \delta \varphi(t), \varphi(t)]}\right\|_{L^{1}(\mathbb{R})}=0,
$$

if $\delta \varphi<0$. Here $\chi_{[a, b]}$ is the characteristic function of the set $[a, b] \subset \mathbb{R}$. To fix ideas we assume that $\delta \varphi(t) \geq 0$ in $t \in[0, T]$. If $\delta \varphi(t) \leq 0$ in $t \in[0, T]$ or if it changes the sign in this interval we argue in a similar way on each time interval where the sign of $\delta \varphi(t)$ is preserved. In step 2 we prove the boundedness of the sequence

$$
\frac{1}{\varepsilon}\left(u_{\varepsilon}(\cdot, t)-u(\cdot, t)-\varepsilon \delta u(\cdot, t)+[u]_{\varphi(t)} \chi_{[\varphi(t), \varphi(t)+\varepsilon \delta \varphi(t)]}(\cdot)\right)
$$

in $L^{1}(\mathbb{R})$, uniformly in $t \in[0, T]$. In step 3 we identify the weak limit as zero. In step 4 we prove that the convergence is strong in $L^{1}(\mathbb{R})$. Finally, in step 5 we prove some identities that are assumed to hold in the previous steps.

Step 1. We prove that for all $t \in[0, T]$ the path $\left(u_{\varepsilon}(\cdot, t), \varphi_{\varepsilon}(t)\right)$ is a regular variation for $(u(\cdot, t), \varphi(t))$. Since $(u, \varphi)$ satisfies (4.24) we may assume that there exists $\varepsilon_{0}>0$ such that for $\varepsilon<\varepsilon_{0}$ the function $u_{\varepsilon}$ is Lipschitz continuous in $\mathbb{R} \times[0, T]$ with one single discontinuity at $x=\varphi_{\varepsilon}(t)$ for $t \in[0, T]$. Moreover, from (2.3), we have

$$
\left\|u_{\varepsilon}-u\right\|_{L^{1}(\mathbb{R})} \leq t \varepsilon\|\delta f\|_{L i p}\left\|u^{0}\right\|_{B V(\mathbb{R})} .
$$

This means, in particular, that $u_{\varepsilon}(x, t) \rightarrow u(x, t)$ pointwise; i.e., for all $(x, t)$ outside the discontinuity $\Sigma$, and

$$
\left|\varphi_{\varepsilon}(t)-\varphi(t)\right| \leq C \varepsilon, \quad \text { as } \varepsilon \rightarrow 0 \text { for all } t \in[0, T],
$$

for some constant $C>0$. Moreover, for each $t \in[0, T]$, to both sides of the discontinuity $x=\varphi_{\varepsilon}(t)$, the Lipschitz constant for $u_{\varepsilon}$ is uniform in $\varepsilon$. This is easily obtained adapting the argument in the proof of (4.13) above. This proves that $u_{\varepsilon}$ is a regular variation in the sense of Definition 4.1

Step 2. Boundedness of the sequence in (9.2), in the $L^{1}(\mathbb{R})$-norm. We first focus on the region where both $u$ and $u_{\varepsilon}$ are Lipschitz continuous and the estimates can be obtained using the characteristics of $u$. For each $\varepsilon>0$ and $t \in[0, T]$, let us 
introduce the following subsets:

$$
\begin{aligned}
& Q_{\varepsilon, t}^{-}=\left\{x \in \mathbb{R} \text { s.t. } x<\min \left\{\varphi(t), \varphi_{\varepsilon}(t)\right\}\right\} \\
& Q_{\varepsilon, t}^{+}=\left\{x \in \mathbb{R} \text { s.t. } x>\max \left\{\varphi(t), \varphi_{\varepsilon}(t)\right\}\right\} \\
& Q_{\varepsilon, t}^{0}=\left\{x \in \mathbb{R} \text { s.t. } \min \left\{\varphi(t), \varphi_{\varepsilon}(t)\right\} \leq x \leq \max \left\{\varphi(t), \varphi_{\varepsilon}(t)\right\}\right\} .
\end{aligned}
$$

We prove that

$$
\begin{aligned}
& \frac{1}{\varepsilon}\left\|u_{\varepsilon}(\cdot, t)-u(\cdot, t)\right\|_{L^{\infty}\left(Q_{\varepsilon, t}^{-} \cup Q_{\varepsilon, t}^{+}\right)} \leq C, \\
& \lim _{\varepsilon \rightarrow 0}\left\|\partial_{x} u_{\varepsilon}(\cdot, t)-\partial_{x} u(\cdot, t)\right\|_{L^{1}\left(Q_{\varepsilon, t}^{-} \cup Q_{\varepsilon, t}^{+}\right)}=0, \\
& \lim _{\varepsilon \rightarrow 0} \frac{1}{\varepsilon}\left\|u_{\varepsilon}(\cdot, t)-u(\cdot, t)-\varepsilon \delta u(\cdot, t)\right\|_{L^{1}\left(Q_{\varepsilon, t}^{-} \cup Q_{\varepsilon, t}^{+}\right)}=0 .
\end{aligned}
$$

We first consider the bound in (9.4). To fix ideas we focus on $x \in Q_{\varepsilon, t}^{+}$but the same will be true for $Q_{\varepsilon, t}^{-}$with analogous arguments. We define

$$
y(x, t)=x-\left(t-t_{0}\right) f^{\prime}(u(x, t)), \quad y_{\varepsilon}(x, t)=x-\left(t-t_{0}\right) f_{\varepsilon}^{\prime}\left(u_{\varepsilon}(x, t)\right) .
$$

Then,

$$
\begin{aligned}
& \frac{1}{\varepsilon}\left|u_{\varepsilon}(x, t)-u(x, t)\right|=\frac{1}{\varepsilon}\left|u_{\varepsilon}\left(y_{\varepsilon}(x, t), t_{0}\right)-u\left(y(x, t), t_{0}\right)\right| \\
& \leq \frac{1}{\varepsilon}\left|u_{\varepsilon}\left(y_{\varepsilon}(x, t), t_{0}\right)-u\left(y_{\varepsilon}(x, t), t_{0}\right)\right| \\
&+\frac{1}{\varepsilon}\left\|u\left(\cdot, t_{0}\right)\right\|_{L i p}\left(t-t_{0}\right)\left|f_{\varepsilon}^{\prime}\left(u_{\varepsilon}(x, t)\right)-f^{\prime}(u(x, t))\right| \\
& \leq \frac{1}{\varepsilon}\left\|u_{\varepsilon}\left(\cdot, t_{0}\right)-u\left(\cdot, t_{0}\right)\right\|_{L^{\infty}\left(Q_{\varepsilon, t}^{+}\right)} \\
&+\frac{1}{\varepsilon}\left\|u\left(\cdot, t_{0}\right)\right\|_{L i p}\left(t-t_{0}\right)\left(\left|f^{\prime}\left(u_{\varepsilon}(x, t)\right)-f^{\prime}(u(x, t))\right|+\varepsilon\left\|\delta f^{\prime}\right\|_{L^{\infty}}\right) \\
& \leq \frac{1}{\varepsilon}\left\|u_{\varepsilon}\left(\cdot, t_{0}\right)-u\left(\cdot, t_{0}\right)\right\|_{L^{\infty}\left(Q_{\varepsilon, t}^{+}\right)} \\
&+\frac{1}{\varepsilon}\left\|u\left(\cdot, t_{0}\right)\right\|_{L i p}\left(t-t_{0}\right)\left(\left\|f^{\prime \prime}\right\|_{L^{\infty}}\left\|u_{\varepsilon}(\cdot, t)-u(\cdot, t)\right\|_{L^{\infty}\left(Q_{\varepsilon, t}^{+}\right)}+\varepsilon\left\|\delta f^{\prime}\right\|_{L^{\infty}}\right) .
\end{aligned}
$$

Here, $\left\|u\left(\cdot, t_{0}\right)\right\|_{L i p}$ stands for the Lipschitz norm of $u\left(\cdot, t_{0}\right)$ when $x>\varphi\left(t_{0}\right)$, which is uniformly bounded in $t_{0} \in[0, T]$ by hypothesis. Therefore, if we choose $t$ such that $\left(t-t_{0}\right)<\left\|u\left(\cdot, t_{0}\right)\right\|_{L i p}^{-1}\left\|f^{\prime \prime}\right\|_{L^{\infty}}^{-1}$,

$$
\begin{aligned}
\frac{1}{\varepsilon}\left\|u_{\varepsilon}(\cdot, t)-u(\cdot, t)\right\|_{L^{\infty}\left(Q_{\varepsilon, t}^{+}\right) \leq} \leq & \frac{\frac{1}{\varepsilon}\left\|u_{\varepsilon}\left(\cdot, t_{0}\right)-u\left(\cdot, t_{0}\right)\right\|_{L^{\infty}\left(Q_{\varepsilon, t_{0}}^{+}\right)}}{1-\left(t-t_{0}\right)\left\|u\left(\cdot, t_{0}\right)\right\|_{L i p}\left\|f^{\prime \prime}\right\|_{L^{\infty}}} \\
& +\left\|u\left(\cdot, t_{0}\right)\right\|_{L i p}\left(t-t_{0}\right)\left\|\delta f^{\prime}\right\|_{L^{\infty}} .
\end{aligned}
$$

In particular, for $t_{0}=0$, this establishes the estimate (9.4) for $t \in\left[0, t_{1}\right]$ with $t_{1}<\left\|u^{0}\right\|_{L i p}^{-1}\left\|f^{\prime \prime}\right\|_{L^{\infty}}^{-1}$. Then, we can apply (9.8) for $t_{0}=t_{1}$ to obtain the estimate in $t \in\left[t_{1}, 2 t_{1}\right]$ and so on until covering the whole interval $[0, T]$.

Concerning the limit (9.5) we observe that, as before, it is enough to obtain it for a sufficiently small time interval and then combine it with an iterative argument 
in time. For $0 \leq t_{0} \leq t \leq T$, we have,

$$
\begin{aligned}
& \int_{Q_{\varepsilon, t}^{+}}\left|\partial_{x} u_{\varepsilon}(x, t)-\partial_{x} u(x, t)\right| d x \\
& =\int_{Q_{\varepsilon, t}^{+}}\left|\partial_{x}\left(u_{\varepsilon}\left(y_{\varepsilon}(x, t), t_{0}\right)\right)-\partial_{x}\left(u\left(y(x, t), t_{0}\right)\right)\right| d x \\
& \leq \int_{Q_{\varepsilon, t}^{+}}\left|\partial_{x} u_{\varepsilon}\left(y_{\varepsilon}(x, t), t_{0}\right)\right|\left(t-t_{0}\right) \mid\left(f^{\prime \prime}\left(u_{\varepsilon}\right) \partial_{x} u_{\varepsilon}+\varepsilon \delta f^{\prime \prime}\left(u_{\varepsilon}\right) \partial_{x} u_{\varepsilon}-f^{\prime \prime}(u) \partial_{x} u \mid d x\right. \\
& +\int_{Q_{\varepsilon, t}^{+}}\left|\partial_{x} u_{\varepsilon}\left(y_{\varepsilon}(x, t), t_{0}\right)-\partial_{x} u\left(y(x, t), t_{0}\right) \| 1-\left(t-t_{0}\right) f^{\prime \prime}(u) \partial_{x} u\right| d x \\
& \leq\left(\varepsilon\left\|\delta f^{\prime \prime}\right\|_{L^{\infty}}+\left\|f^{\prime \prime}\left(u_{\varepsilon}\right) \partial_{x} u_{\varepsilon}-f^{\prime \prime}(u) \partial_{x} u\right\|_{L^{1}\left(Q_{\varepsilon, t}^{+}\right)}\right)\left\|u_{\varepsilon}(\cdot, t)\right\|_{L i p}\left(t-t_{0}\right) \\
& +C \int_{Q_{\varepsilon, t}^{+}}\left|\partial_{x} u_{\varepsilon}\left(y_{\varepsilon}(x, t), t_{0}\right)-\partial_{x} u\left(y_{\varepsilon}(x, t), t_{0}\right)\right| d x \\
& +C \int_{Q_{\varepsilon, t}^{+}}\left|\partial_{x} u\left(y_{\varepsilon}(x, t), t_{0}\right)-\partial_{x} u\left(y(x, t), t_{0}\right)\right| d x \\
& \leq\left(\varepsilon f^{\prime \prime}\left\|_{L^{\infty}}+\right\| f^{\prime \prime}\left\|_{L^{\infty}}\right\| \partial_{x} u_{\varepsilon}-\partial_{x} u \|_{L^{1}\left(Q_{\varepsilon, t}^{+}\right)}\right)\left\|u_{\varepsilon}\right\|_{L i p}\left(t-t_{0}\right) \\
& +\left(\left\|f^{\prime \prime}\left(u_{\varepsilon}(\cdot, t)\right)-f^{\prime \prime}(u(\cdot, t))\right\|_{L^{\infty}}\|u\|_{B V}\right)\left\|u_{\varepsilon}\right\|_{L i p}\left(t-t_{0}\right) \\
& +C \int_{Q_{\varepsilon, t_{0}}^{+}} \frac{\left|\partial_{y} u_{\varepsilon}\left(y, t_{0}\right)-\partial_{y} u\left(y, t_{0}\right)\right|}{\left(1-\left(t-t_{0}\right)\left\|f_{\varepsilon}^{\prime \prime}\right\|_{L^{\infty}}\left\|u_{\varepsilon}\right\|_{L i p}\right)} d y \\
& +C \int_{Q_{\varepsilon, t}^{+}}\left|\partial_{x} u\left(y_{\varepsilon}(x, t), t_{0}\right)-\partial_{x} u\left(y(x, t), t_{0}\right)\right| d x
\end{aligned}
$$

with $C=1+\left(t-t_{0}\right)\left\|f^{\prime \prime}\right\|_{L^{\infty}}\|u\|_{L i p}$. Thus, if we choose $t$ such that $\left(t-t_{0}\right)<$ $\min _{0<\varepsilon<\varepsilon_{0}}\left\|u_{\varepsilon}\right\|_{L i p}^{-1}\left\|f^{\prime \prime}\right\|_{L^{\infty}}^{-1}$, then

$$
\begin{aligned}
& \lim _{\varepsilon \rightarrow 0} \int_{Q_{\varepsilon, t}^{+}}\left|\partial_{x} u_{\varepsilon}(x, t)-\partial_{x} u(x, t)\right| d x \leq C_{1} \lim _{\varepsilon \rightarrow 0}\left\|f^{\prime \prime}\left(u_{\varepsilon}\right)-f^{\prime \prime}(u)\right\|_{L^{\infty}\left(Q_{\varepsilon, t}^{+}\right)} \\
& +C_{2} \lim _{\varepsilon \rightarrow 0} \int_{Q_{\varepsilon, t}^{+}}\left|\partial_{x} u\left(x-\left(t-t_{0}\right) f_{\varepsilon}^{\prime}\left(u_{\varepsilon}\right), t_{0}\right)-\partial_{x} u\left(x-\left(t-t_{0}\right) f^{\prime}(u), t_{0}\right)\right| d x \\
& +C_{3} \lim _{\varepsilon \rightarrow 0} \int_{Q_{\varepsilon, t_{0}}^{+}}\left|\partial_{x} u_{\varepsilon}\left(x, t_{0}\right)-\partial_{x} u\left(x, t_{0}\right)\right| d x
\end{aligned}
$$

for some constants $C_{1}, C_{2}, C_{3}$ which only depend on $\|u\|_{L i p},\left\|f^{\prime \prime}\right\|_{L^{\infty}},\left\|u_{\varepsilon}\right\|_{\text {Lip }}$, $\left\|f_{\varepsilon}^{\prime \prime}\right\|_{L^{\infty}}$ and $\left(t-t_{0}\right)$, which are quantities that do not depend on $\varepsilon$. Now, we show that the first two limits on the right-hand side of (9.9) are zero. The last limit is obviously zero for $t_{0}=0$ and, for larger time, we can apply the iterative argument described before. The first limit on the right-hand side is zero as a consequence of the uniform convergence of $f^{\prime \prime}\left(u_{\varepsilon}(\cdot, t)\right)$ to $f^{\prime \prime}(u(\cdot, t))$ as $\varepsilon \rightarrow 0$, which is due to the uniform continuity of $f^{\prime \prime}(s)$ in the compact set $s \in\left[-\left\|u^{0}\right\|,\left\|u^{0}\right\|\right]$ and the estimate in (9.4).

The second limit on the right-hand side of (9.9) is obtained by a density argument. Since $\partial_{x} u\left(\cdot, t_{0}\right) \in L^{1}\left(\varphi\left(t_{0}\right), \infty\right)$ there exists a sequence $h_{n}(x) \in C_{0}^{1}\left(\varphi\left(t_{0}\right), \infty\right)$ 
such that $h_{n} \rightarrow \partial_{x} u\left(\cdot, t_{0}\right)$ in $L^{1}\left(\varphi\left(t_{0}\right), \infty\right)$. Then

$$
\begin{aligned}
& \int_{Q_{\varepsilon, t}^{+}}\left|\partial_{x} u\left(x-\left(t-t_{0}\right) f_{\varepsilon}^{\prime}\left(u_{\varepsilon}\right), t_{0}\right)-\partial_{x} u\left(x-\left(t-t_{0}\right) f^{\prime}(u), t_{0}\right)\right| d x \\
& \leq \int_{Q_{\varepsilon, t}^{+}}\left|\partial_{x} u\left(x-\left(t-t_{0}\right) f_{\varepsilon}^{\prime}\left(u_{\varepsilon}\right), t_{0}\right)-h_{n}\left(x-\left(t-t_{0}\right) f_{\varepsilon}^{\prime}\left(u_{\varepsilon}\right), t_{0}\right)\right| d x \\
& \quad+\int_{Q_{\varepsilon, t}^{+}}\left|h_{n}\left(x-\left(t-t_{0}\right) f_{\varepsilon}^{\prime}\left(u_{\varepsilon}\right), t_{0}\right)-h_{n}\left(x-\left(t-t_{0}\right) f^{\prime}(u), t_{0}\right)\right| d x \\
& \quad+\int_{Q_{\varepsilon, t}^{+}}\left|h_{n}\left(x-\left(t-t_{0}\right) f^{\prime}(u), t_{0}\right)-\partial_{x} u\left(x-\left(t-t_{0}\right) f^{\prime}(u), t_{0}\right)\right| d x .
\end{aligned}
$$

Thus, it suffices to see that the first and third limits on the right-hand side converge to zero as $n \rightarrow \infty$, uniformly in $\varepsilon$, while the second limit converges to zero for each $h_{n}$. The convergence of this second term is obvious since $h_{n}$ is smooth and,

$$
\left|f_{\varepsilon}^{\prime}\left(u_{\varepsilon}\right)-f^{\prime}(u)\right| \leq\left\|f^{\prime \prime}\right\|_{L^{\infty}}\left\|u_{\varepsilon}(\cdot, t)-u(\cdot, t)\right\|_{L^{\infty}\left(Q_{\varepsilon, t}^{+}\right)} \leq C \varepsilon .
$$

Concerning the first term in (9.10) we observe that, after the change of variables $y=x-\left(t-t_{0}\right) f_{\varepsilon}^{\prime}\left(u_{\varepsilon}(x, t)\right)$, we obtain

$$
\begin{aligned}
& \int_{Q_{\varepsilon, t}^{+}}\left|\partial_{x} u\left(x-\left(t-t_{0}\right) f_{\varepsilon}^{\prime}\left(u_{\varepsilon}\right), t_{0}\right)-h_{n}\left(x-\left(t-t_{0}\right) f_{\varepsilon}^{\prime}\left(u_{\varepsilon}\right), t_{0}\right)\right| d x \\
& \quad \leq \int_{Q_{\varepsilon, t_{0}}^{+}}\left|\partial_{x} u\left(y, t_{0}\right)-h_{n}\left(y, t_{0}\right)\right|\left(1-\left(t-t_{0}\right)\left\|f_{\varepsilon}^{\prime \prime}\right\|_{L^{\infty}}\left\|u_{\varepsilon}\right\|_{L i p}\right)^{-1} d y \\
& \quad \leq\left(1-\left(t-t_{0}\right)\left\|f_{\varepsilon}^{\prime \prime}\right\|_{L^{\infty}}\left\|u_{\varepsilon}\right\|_{L i p}\right)^{-1} \int_{\varphi\left(t_{0}\right)}^{\infty}\left|\partial_{x} u\left(y, t_{0}\right)-h_{n}\left(y, t_{0}\right)\right| d y
\end{aligned}
$$

which converges to zero, uniformly in $\varepsilon$, as $n \rightarrow \infty$, when $\left(t-t_{0}\right)<\left\|u_{\varepsilon}\right\|_{L i p}^{-1}\left\|f_{\varepsilon}^{\prime \prime}\right\|_{L^{\infty}}^{-1}$. Recall that both $\left\|u_{\varepsilon}\right\|_{L i p}$ and $\left\|f_{\varepsilon}^{\prime \prime}\right\|_{L^{\infty}}$ are uniformly bounded in $\varepsilon$, when $x \in Q_{\varepsilon, t}^{+}$. Similarly, the third term in the right-hand side of (9.10) tends to zero, uniformly in $\varepsilon$, as $h \rightarrow \infty$.

We consider now the limit in (9.6). Let us define

$$
w_{\varepsilon}=\frac{1}{\varepsilon}\left(u_{\varepsilon}-u-\varepsilon \delta u\right), \quad \text { in } t \in[0, T], x>\max \left\{\varphi(t), \varphi_{\varepsilon}(t)\right\} .
$$

Note that $w_{\varepsilon}$ is a Lipschitz continuous solution of the system,

$$
\left\{\begin{array}{l}
\partial_{t} w_{\varepsilon}+\partial_{x}\left(f^{\prime}(u) w_{\varepsilon}\right)=-\partial_{x}\left(\delta f\left(u_{\varepsilon}\right)-\delta f(u)\right) \\
-\frac{1}{\varepsilon} \partial_{x}\left(f\left(u_{\varepsilon}\right)-f(u)-f^{\prime}(u)\left(u_{\varepsilon}-u\right)\right), \quad t \in[0, T], x>\max \left\{\varphi(t), \varphi_{\varepsilon}(t)\right\} \\
w_{\varepsilon}(x, 0)=0, \quad x>\varphi^{0} .
\end{array}\right.
$$

System (9.11) is analogous to the linearized system (4.10) for which the solution is given by (4.12). Thus, the solution of (9.11) is given by,

$$
w_{\varepsilon}=-t \partial_{x}\left(\delta f\left(u_{\varepsilon}\right)-\delta f(u)\right)-\frac{t}{\varepsilon} \partial_{x}\left(f\left(u_{\varepsilon}\right)-f(u)-f^{\prime}(u)\left(u_{\varepsilon}-u\right)\right) .
$$


Then,

$$
\begin{aligned}
& \int_{Q_{\varepsilon, t}^{+}}\left|w_{\varepsilon}(x, t)\right| d x \leq t \int_{Q_{\varepsilon, t}^{+}}\left|\delta f^{\prime}\left(u_{\varepsilon}\right) \partial_{x} u_{\varepsilon}-\delta f^{\prime}(u) \partial_{x} u\right| d x \\
& +\frac{t}{\varepsilon} \int_{Q_{\varepsilon, t}^{+}}\left|f^{\prime}\left(u_{\varepsilon}\right) \partial_{x} u_{\varepsilon}-f^{\prime}(u) \partial_{x} u-f^{\prime \prime}(u) \partial_{x} u\left(u_{\varepsilon}-u\right)-f^{\prime}(u)\left(\partial_{x} u_{\varepsilon}-\partial_{x} u\right)\right| d x \\
& \leq t \int_{Q_{\varepsilon, t}^{+}}\left|\delta f^{\prime}\left(u_{\varepsilon}\right)-\delta f^{\prime}(u)\left\|\partial_{x} u\left|d x+t \int_{Q_{\varepsilon, t}^{+}}\right| \delta f^{\prime}(u)\right\| \partial_{x} u_{\varepsilon}-\partial_{x} u\right| d x \\
& \quad+\frac{t}{\varepsilon} \int_{Q_{\varepsilon, t}^{+}}\left|f^{\prime}\left(u_{\varepsilon}\right)-f^{\prime}(u)-f^{\prime \prime}(u)\left(u_{\varepsilon}-u\right) \| \partial_{x} u\right| d x \\
& \quad+\frac{t}{\varepsilon} \int_{Q_{\varepsilon, t}^{+}}\left|f^{\prime}\left(u_{\varepsilon}\right)-f^{\prime}(u) \| \partial_{x} u_{\varepsilon}-\partial_{x} u\right| d x \\
& \leq t \\
& \quad\left\|\delta f^{\prime \prime}\right\|_{L^{\infty}}\|u\|_{L i p} \int_{Q_{\varepsilon, t}^{+}}\left|u_{\varepsilon}-u\right| d x+t\left\|\delta f^{\prime}\right\|_{L^{\infty}} \int_{Q_{\varepsilon, t}^{+}}\left|\partial_{x} u_{\varepsilon}-\partial_{x} u\right| d x \\
& \quad+\frac{t}{\varepsilon}\|u\|_{B V}\left\|f^{\prime}\left(u_{\varepsilon}\right)-f^{\prime}(u)-f^{\prime \prime}(u)\left(u_{\varepsilon}-u\right)\right\|_{L^{\infty}} \\
& \quad+\frac{t}{\varepsilon}\left\|f^{\prime \prime}\right\|_{L^{\infty}}\left\|u_{\varepsilon}-u\right\|_{L^{\infty}} \int_{Q_{\varepsilon, t}^{+}}\left|\partial_{x} u_{\varepsilon}-\partial_{x} u\right| d x
\end{aligned}
$$

which converges to zero, as $\varepsilon \rightarrow 0$, in view of (9.4)-(9.5).

Once (9.4)-(9.6) are proved we address the boundedness of the sequence in (9.2). We have,

$$
\begin{aligned}
\frac{1}{\varepsilon}\left\|u_{\varepsilon}-u-\varepsilon \delta u+[u]_{\varphi(t)} \chi_{[\varphi, \varphi+\varepsilon \delta \varphi]}\right\|_{L^{1}(\mathbb{R})} \\
=\frac{1}{\varepsilon}\left\|u_{\varepsilon}-u-\varepsilon \delta u+[u]_{\varphi(t)} \chi_{[\varphi, \varphi+\varepsilon \delta \varphi]}\right\|_{L^{1}\left(Q_{\varepsilon, t}^{-} \cup Q_{\varepsilon, t}^{+}\right)} \\
\quad+\frac{1}{\varepsilon}\left\|u_{\varepsilon}-u-\varepsilon \delta u+[u]_{\varphi(t)} \chi_{[\varphi, \varphi+\varepsilon \delta \varphi]}\right\|_{L^{1}\left(Q_{\varepsilon, t}^{0}\right)} \\
\leq \frac{1}{\varepsilon}\left\|u_{\varepsilon}-u-\varepsilon \delta u\right\|_{L^{1}\left(Q_{\varepsilon, t}^{-} \cup Q_{\varepsilon, t}^{+}\right)}+\frac{[u]_{\varphi(t)}}{\varepsilon}\left\|\chi_{[\varphi, \varphi+\varepsilon \delta \varphi]}\right\|_{L^{1}\left(Q_{\varepsilon, t}^{-} \cup Q_{\varepsilon, t}^{+}\right)} \\
\quad+\frac{1}{\varepsilon}\left\|u_{\varepsilon}-u-\varepsilon \delta u+[u]_{\varphi(t)} \chi_{[\varphi, \varphi+\varepsilon \delta \varphi]}\right\|_{L^{1}\left(Q_{\varepsilon, t}^{0}\right)} .
\end{aligned}
$$

Here, the first term on the right-hand side is bounded, as $\varepsilon \rightarrow 0$, due to (9.6), the second one is bounded since the support of the function inside the $L^{1}$-norm is of the order of $\varepsilon$ and the third one is bounded since the function inside the $L^{1}$-norm is uniformly bounded in $L^{\infty}$ and the measure of $Q_{\varepsilon, t}^{0}$ is of the order of $\varepsilon$, uniformly in $t$, due to (9.3) together with the definition of $Q_{\varepsilon, t}^{0}$.

Step 3. Identification of the weak limit of (9.2). From the previous step we deduce that the sequence in (9.2) converges, as $\varepsilon \rightarrow 0$, weakly in $L^{1}$, at least for a subsequence that we still denote $\varepsilon$. To identify the limit we focus on the weak 
formulations of $u_{\varepsilon}$ and $u$. We have,

$$
\begin{aligned}
0= & \frac{1}{\varepsilon} \iint_{\mathbb{R} \times(0, T)}\left(\left(u_{\varepsilon}-u\right) \partial_{t} \psi+\left(f_{\varepsilon}\left(u_{\varepsilon}\right)-f(u)\right) \partial_{x} \psi\right) d x d t \\
= & \frac{1}{\varepsilon} \iint_{\mathbb{R} \times(0, T)}\left(u_{\varepsilon}-u-\varepsilon \delta u+[u]_{\varphi(t)} \chi_{[\varphi, \varphi+\varepsilon \delta \varphi]}\right) \partial_{t} \psi d x d t \\
& -\frac{1}{\varepsilon} \int_{0}^{T}[f(u)]_{\varphi} \partial_{x} \psi(\varphi, t)\left(\varphi_{\varepsilon}-\varphi-\varepsilon \delta \varphi\right) d t \\
& +\frac{1}{\varepsilon} \iint_{\mathbb{R} \times(0, T)}\left(\varepsilon \delta u-[u]_{\varphi(t)} \chi_{[\varphi, \varphi+\varepsilon \delta \varphi]}\right) \partial_{t} \psi d x d t \\
& +\frac{1}{\varepsilon} \iint_{\mathbb{R} \times(0, T)}\left(f_{\varepsilon}\left(u_{\varepsilon}\right)-f(u)\right) \partial_{x} \psi d x d t \\
& +\frac{1}{\varepsilon} \int_{0}^{T}[f(u)]_{\varphi} \partial_{x} \psi(\varphi, t)\left(\varphi_{\varepsilon}-\varphi-\varepsilon \delta \varphi\right) d t,
\end{aligned}
$$

for all $\psi \in C_{0}^{1}(\mathbb{R} \times[0, T))$, the set of $C^{1}$ functions with compact support. Assume for the moment that the last three terms in the right-hand side of (9.12) tend to zero as $\varepsilon \rightarrow 0$, i.e.,

$$
\begin{aligned}
0= & \lim _{\varepsilon \rightarrow 0}\left\{\frac{1}{\varepsilon} \iint_{\mathbb{R} \times(0, T)}\left(\varepsilon \delta u-[u]_{\varphi(t)} \chi_{[\varphi, \varphi+\varepsilon \delta \varphi]}\right) \partial_{t} \psi d x d t\right. \\
& +\frac{1}{\varepsilon} \iint_{\mathbb{R} \times(0, T)}\left(f_{\varepsilon}\left(u_{\varepsilon}\right)-f(u)\right) \partial_{x} \psi d x d t \\
& \left.+\frac{1}{\varepsilon} \int_{0}^{T}[f(u)]_{\varphi} \partial_{x} \psi(\varphi, t)\left(\varphi_{\varepsilon}-\varphi-\varepsilon \delta \varphi\right) d t\right\} .
\end{aligned}
$$

Then, if we consider particular test functions $\psi(x, t)$ with support in the following neighbourhood of $\Sigma$,

$$
\left\{(x, t) \text { such that }|x-\varphi(t)|<\varepsilon^{2}, \quad 0 \leq t \leq T\right\},
$$

the first term in the right-hand side of (9.12) vanishes as $\varepsilon \rightarrow 0$ since the integrand is bounded by $C t e / \varepsilon$ and the support of the integral is a region of measure $\varepsilon^{2} C t e$, as $\varepsilon \rightarrow 0$. Therefore,

$$
\lim _{\varepsilon \rightarrow 0} \frac{1}{\varepsilon} \int_{0}^{T}[f(u)]_{\varphi} \partial_{x} \psi(\varphi, t)\left(\varphi_{\varepsilon}-\varphi-\varepsilon \delta \varphi\right) d t=0 .
$$

This provides the convergence,

$$
[f(u)]_{\varphi} \frac{1}{\varepsilon}\left(\varphi_{\varepsilon}-\varphi-\varepsilon \delta \varphi\right) \rightarrow 0,
$$

at least in the sense of distributions.

From (9.12), 9.13) and (9.15) we obtain the convergence of the first term of (9.12), for any test function $\psi$. Now, if we consider $\psi(x, t)=\psi_{1}(t) \psi_{2}(x)$ in separate variables in formula (9.12), then

$$
\begin{aligned}
0 & =\lim _{\varepsilon \rightarrow 0} \frac{1}{\varepsilon} \int_{0}^{T} \int_{\mathbb{R}}\left(u_{\varepsilon}-u-\varepsilon \delta u+[u]_{\varphi(t)} \chi_{[\varphi, \varphi+\varepsilon \delta \varphi]}\right) \psi_{1}^{\prime} \psi_{2} d x d t \\
& =\int_{0}^{T} \psi_{1}^{\prime} \lim _{\varepsilon \rightarrow 0} \frac{1}{\varepsilon} \int_{\mathbb{R}}\left(u_{\varepsilon}-u-\varepsilon \delta u+[u]_{\varphi(t)} \chi_{[\varphi, \varphi+\varepsilon \delta \varphi]}\right) \psi_{2} d x d t
\end{aligned}
$$


where we have used the dominated convergence theorem. Note that the integrand of the time integral is uniformly bounded in $t \in[0, T]$ since the bound for (9.2) obtained in the step 2 above can be chosen independent of $t$. Therefore we obtain,

$$
\lim _{\varepsilon \rightarrow 0} \frac{1}{\varepsilon} \int_{\mathbb{R}}\left(u_{\varepsilon}-u-\varepsilon \delta u+[u]_{\varphi(t)} \chi_{[\varphi, \varphi+\varepsilon \delta \varphi]}\right) \psi_{x} d x=0 \text {, a.e. } t \in(0, T),
$$

for all $\psi_{x} \in C_{0}^{1}(\mathbb{R})$. In fact, the same is true for $\psi_{x} \in L^{\infty}(\mathbb{R})$ with compact support, since $\varepsilon^{-1}\left(u_{\varepsilon}-u-\varepsilon \delta u+[u]_{\varphi(t)} \chi_{[\varphi, \varphi+\varepsilon \delta \varphi]}\right)$ is bounded in $L^{1}(\mathbb{R})$ for each $t \in[0, T]$, as $\varepsilon \rightarrow 0$. This shows the weak convergence

$$
\varepsilon^{-1}\left(u_{\varepsilon}(\cdot, t)-u(\cdot, t)-\varepsilon \delta u(\cdot, t)+[u]_{\varphi(t)} \chi_{[\varphi(t), \varphi(t)+\varepsilon \delta \varphi(t)]}(\cdot)\right) \rightarrow 0 \text { weakly in } L^{1}(\mathbb{R}) .
$$

Step 4. Strong convergence of (9.2). In order to prove the strong convergence in $L^{1}$ it suffices to see that the convergence in measure holds, for any measurable subset $B \subset \mathbb{R}$ with finite measure (see for example [36], p. 122). This means that, for any $\delta>0$, the measure of the subset,

$$
\left\{x \in B \text { with }\left|\varepsilon^{-1}\left(u_{\varepsilon}(x, t)-u(x, t)-\varepsilon \delta u(x, t)+[u]_{\varphi(t)} \chi_{[\varphi(t), \varphi(t)+\varepsilon \delta \varphi]}(x)\right)\right|>\delta\right\}
$$

converges to zero, as $\varepsilon \rightarrow 0$. Recall that, as we stated in (9.6), in the region $Q_{\varepsilon, t}^{-} \cup Q_{\varepsilon, t}^{+}, \varepsilon^{-1}\left(u_{\varepsilon}(x, t)-u(x, t)-\varepsilon \delta u(x, t)\right)$ converges strongly in $L^{1}$. Thus, the subset in (9.18) is inside the region $Q_{\varepsilon, t}^{0} \cup[\varphi(t), \varphi(t)+\varepsilon \delta \varphi(t)]$. But the measure of this last subset converges to zero as $\varepsilon \rightarrow 0$, uniformly in $t \in[0, T]$, and therefore the convergence in measure holds. This proves (9.1).

Step 5. It remains to check that formula (9.13) holds true. Observe that it suffices to prove the following two identities:

$$
\begin{aligned}
& \lim _{\varepsilon \rightarrow 0} \frac{1}{\varepsilon} \int \int_{\mathbb{R} \times(0, T)}\left(-[u]_{\varphi(t)} \chi_{[\varphi, \varphi+\varepsilon \delta \varphi]}\right) \partial_{t} \psi d x d t \\
&-\int_{0}^{T}[u]_{\varphi(t)} \varphi^{\prime}(t) \delta \varphi(t) \partial_{x} \psi(\varphi(t), t) d t \\
&(9.19)= \int_{0}^{T}\left(-\left[\partial_{x} f(u)\right]_{\varphi(t)} \delta \varphi(t)+\varphi^{\prime}\left[\partial_{x} u\right]_{\varphi(t)} \delta \varphi(t)+[u]_{\varphi(t)} \delta \varphi^{\prime}(t)\right) \psi(\varphi(t), t) d t, \\
& \lim _{\varepsilon \rightarrow 0} \frac{1}{\varepsilon}\left\{\iint_{\mathbb{R} \times(0, T)}\left(f_{\varepsilon}\left(u_{\varepsilon}\right)-f(u)\right) \partial_{x} \psi d x d t+\int_{0}^{T}[f(u)]_{\varphi} \partial_{x} \psi(\varphi, t)\left(\varphi_{\varepsilon}-\varphi\right) d t\right\} \\
&(9.20)=\iint_{\mathbb{R} \times(0, T)}\left(f^{\prime}(u) \delta u+\delta f(u)\right) \partial_{x} \psi .
\end{aligned}
$$

In fact, if (9.19)-(9.20) hold, then (9.13) is equivalent to

$$
\begin{aligned}
0= & \iint_{\mathbb{R} \times(0, T)}\left(f^{\prime}(u) \delta u+\delta f(u)\right) \partial_{x} \psi+\iint_{\mathbb{R} \times(0, T)} \delta u \partial_{t} \psi \\
& +\int_{0}^{T}\left(-\left[\partial_{x} f(u)\right]_{\varphi(t)} \delta \varphi(t)+\varphi^{\prime}\left[\partial_{x} u\right]_{\varphi(t)} \delta \varphi(t)+[u]_{\varphi(t)} \delta \varphi^{\prime}(t)\right) \psi(\varphi(t), t) d t
\end{aligned}
$$

which is the weak formulation of the linearized problem (4.27).

We first focus on the limit in (9.19). As we are interested in the limit as $\varepsilon \rightarrow 0$, only first order terms with respect to $\varepsilon$ are required. In the following, we write 
$\mathcal{O}(\varepsilon)$ for any term satisfying

We have

$$
\lim _{\varepsilon \rightarrow 0} \frac{|\mathcal{O}(\varepsilon)|}{\varepsilon} \leq C t e, \text { uniformly in }(x, t) .
$$

$$
\begin{aligned}
\frac{1}{\varepsilon} \iint_{\mathbb{R} \times(0, T)}\left(-[u]_{\varphi(t)} \chi_{[\varphi, \varphi+\varepsilon \delta \varphi]}\right) \partial_{t} \psi d x d t \\
=-\frac{1}{\varepsilon} \int_{0}^{T}[u]_{\varphi(t)} \int_{\varphi(t)}^{\varphi(t)+\varepsilon \delta \varphi(t)} \partial_{t} \psi d t d x \\
=-\frac{1}{\varepsilon} \int_{0}^{T}[u]_{\varphi(t)}\left(\frac{d}{d t} \int_{\varphi(t)}^{\varphi(t)+\varepsilon \delta \varphi(t)} \psi d x\right) d t \\
\quad+\frac{1}{\varepsilon} \int_{0}^{T}[u]_{\varphi(t)}\left(\left(\varphi^{\prime}+\varepsilon \delta \varphi^{\prime}\right) \psi(\varphi+\varepsilon \delta \varphi, t)-\varphi^{\prime} \psi(\varphi, t)\right) d t \\
=\frac{1}{\varepsilon} \int_{0}^{T} \frac{d}{d t}\left([u]_{\varphi(t)}\right) \int_{\varphi(t)}^{\varphi(t)+\varepsilon \delta \varphi(t)} \psi d x d t \\
\quad+\frac{1}{\varepsilon} \int_{0}^{T}[u]_{\varphi(t)}\left(\left(\varphi^{\prime}+\varepsilon \delta \varphi^{\prime}\right) \psi(\varphi+\varepsilon \delta \varphi, t)-\varphi^{\prime} \psi(\varphi, t)\right) d t
\end{aligned}
$$

Note that, in the last identity, the boundary terms coming from the integration by parts vanish since we are assuming $\psi(x, t)$ of compact support in $t \in[0, T)$ and $\delta \varphi(0)=0$. To simplify the first term on the right-hand side we write the jump $[u]_{\varphi(t)}$ in a more explicit way. Let us introduce the following notation:

$$
u\left(x^{+}, t\right)=\lim _{\delta \rightarrow 0, \delta>0} u(x+\delta, t), \quad u\left(x^{-}, t\right)=\lim _{\delta \rightarrow 0, \delta>0} u(x-\delta, t) .
$$

Observe that

$$
\begin{aligned}
& \frac{d}{d t}\left([u]_{\varphi(t)}\right)=\frac{d}{d t}\left(u\left(\varphi(t)^{+}, t\right)-u\left(\varphi(t)^{-}, t\right)\right) \\
& \quad=\partial_{t} u\left(\varphi(t)^{+}, t\right)-\partial_{t} u\left(\varphi(t)^{-}, t\right)+\partial_{x} u\left(\varphi(t)^{+}, t\right) \varphi^{\prime}(t)-\partial_{x} u\left(\varphi(t)^{-}, t\right) \varphi^{\prime}(t) \\
& \quad=\left[\partial_{t} u\right]_{\varphi(t)}+\varphi^{\prime}\left[\partial_{x} u\right]_{\varphi(t)}=-\left[\partial_{x} f(u)\right]_{\varphi(t)}+\varphi^{\prime}\left[\partial_{x} u\right]_{\varphi(t)},
\end{aligned}
$$

where we have used in the last identity that, to both sides of the discontinuity, the function $u$ satisfies the first equation in (4.25).

On the other hand, as $\psi$ is assumed to be smooth, it can be expanded near $x=\varphi(t)$ using the Taylor formula, i.e.,

$$
\psi(\varphi+\varepsilon \delta \varphi, t)=\psi(\varphi, t)+\varepsilon \delta \varphi \partial_{x} \psi(\varphi, t)+\mathcal{O}\left(\varepsilon^{2}\right) .
$$

Therefore, at first order, we have

$$
\frac{1}{\varepsilon} \int_{\varphi(t)}^{\varphi(t)+\varepsilon \delta \varphi(t)} \psi d x d t=\psi(\varphi, t) \delta \varphi(t)+\mathcal{O}(\varepsilon) .
$$

From (9.22)- (9.24) the right-hand side of (9.21) can be written as

$$
\begin{aligned}
& \int_{0}^{T}\left(-\left[\partial_{x} f(u)\right]_{\varphi(t)} \delta \varphi(t)+\varphi^{\prime}\left[\partial_{x} u\right]_{\varphi(t)} \delta \varphi(t)+[u]_{\varphi(t)} \delta \varphi^{\prime}(t)\right) \psi(\varphi(t), t) d t \\
& +\int_{0}^{T}[u]_{\varphi(t)} \varphi^{\prime}(t) \delta \varphi(t) \partial_{x} \psi(\varphi(t), t) d t+\mathcal{O}(\varepsilon),
\end{aligned}
$$

This allows us to deduce the limit in (9.19). 
Concerning the identity (9.20), we have

$$
\begin{aligned}
\lim _{\varepsilon \rightarrow 0} & \frac{1}{\varepsilon} \iint_{\mathbb{R} \times(0, T)}\left(f_{\varepsilon}\left(u_{\varepsilon}\right)-f(u)\right) \partial_{x} \psi d x d t \\
= & \lim _{\varepsilon \rightarrow 0} \frac{1}{\varepsilon} \int_{0}^{T} \int_{Q_{\varepsilon, t}^{-} \cup Q_{\varepsilon, t}^{+}}\left(f\left(u_{\varepsilon}\right)+\varepsilon \delta f\left(u_{\varepsilon}\right)-f(u)\right) \partial_{x} \psi d x d t \\
& +\lim _{\varepsilon \rightarrow 0} \frac{1}{\varepsilon} \iint_{Q_{\varepsilon, t}^{0}}\left(f\left(u_{\varepsilon}\right)+\varepsilon \delta f\left(u_{\varepsilon}\right)-f(u)\right) \partial_{x} \psi d x d t .
\end{aligned}
$$

Observe that, on $Q_{\varepsilon, t}^{-} \cup Q_{\varepsilon, t}^{+}$, the Taylor expansion of both $f$ and $\delta f$ at $u$ give us

$$
\begin{aligned}
f\left(u_{\varepsilon}\right) & =f(u)+f^{\prime}(u)\left(u_{\varepsilon}-u\right)+\mathcal{O}\left(\left\|u_{\varepsilon}-u\right\|_{L^{\infty}}^{2}\right), \\
\delta f\left(u_{\varepsilon}\right) & =\delta f(u)+\mathcal{O}\left(\left\|u_{\varepsilon}-u\right\|_{L^{\infty}}\right) .
\end{aligned}
$$

This, together with the convergence results stated in (9.4)-(9.6), provide

$$
\begin{aligned}
& \lim _{\varepsilon \rightarrow 0} \frac{1}{\varepsilon}\left\|f\left(u_{\varepsilon}\right)-f(u)-\varepsilon f^{\prime}(u) \delta u\right\|_{L^{1}\left(Q_{\varepsilon, t}^{-} \cup Q_{\varepsilon, t}^{+}\right)}=0, \\
& \delta f\left(u_{\varepsilon}\right)=\delta f(u)+\mathcal{O}(\varepsilon) .
\end{aligned}
$$

Therefore, the first limit on the right-hand side of (9.25) can be simplified as follows:

$$
\begin{aligned}
& \lim _{\varepsilon \rightarrow 0} \frac{1}{\varepsilon} \int_{0}^{T} \int_{Q_{\varepsilon, t}^{-} \cup Q_{\varepsilon, t}^{+}}\left(f\left(u_{\varepsilon}\right)+\varepsilon \delta f\left(u_{\varepsilon}\right)-f(u)\right) \partial_{x} \psi d x d t \\
& \quad=\lim _{\varepsilon \rightarrow 0} \iint_{Q_{\varepsilon, t}^{-} \cup Q_{\varepsilon, t}^{+}}\left(f^{\prime}(u) \delta u+\delta f(u)\right) \partial_{x} \psi d x d t \\
& \quad=\iint_{\mathbb{R} \times(0, T)}\left(f^{\prime}(u) \delta u+\delta f(u)\right) \partial_{x} \psi d x d t .
\end{aligned}
$$

Finally, to simplify the last limit in (9.25) we assume, without loss of generality, that $\varphi_{\varepsilon}(t) \geq \varphi(t)$. In this case, in $Q_{\varepsilon, t}^{0}$ we have,

$$
\begin{aligned}
\left\|u_{\varepsilon}(\cdot, t)-u\left(\varphi(t)^{-}, t\right)\right\|_{L^{\infty}\left(Q_{\varepsilon, t}^{0}\right)} \leq & \left\|u_{\varepsilon}(\cdot, t)-u_{\varepsilon}\left(\varphi(t)^{-}, t\right)\right\|_{L^{\infty}\left(Q_{\varepsilon, t}^{0}\right)} \\
& +\left|u_{\varepsilon}\left(\varphi(t)^{-}, t\right)-u\left(\varphi(t)^{-}, t\right)\right| \\
\leq & \left\|u_{\varepsilon}(\cdot, t)\right\|_{L i p}\left|\varphi_{\varepsilon}(t)-\varphi(t)\right|+C \varepsilon=\mathcal{O}(\varepsilon), \\
\left\|u(\cdot, t)-u\left(\varphi(t)^{+}, t\right)\right\|_{L^{\infty}\left(Q_{\varepsilon, t}^{0}\right)} \leq & \|u(\cdot, t)\|_{L i p}\left|\varphi_{\varepsilon}(t)-\varphi(t)\right|=\mathcal{O}(\varepsilon),
\end{aligned}
$$

and

$$
\int_{0}^{T} \int_{Q_{\varepsilon, t}^{0}}|\delta u(x, t)| d x d t \leq T\|\delta u\|_{L^{\infty}} \text { meas }\left(Q_{\varepsilon, t}^{0}\right)=\mathcal{O}(\varepsilon) .
$$

Thus, the last limit in (9.25) can be simplified as follows:

$$
\begin{aligned}
& \frac{1}{\varepsilon} \int_{0}^{T} \int_{Q_{\varepsilon, t}^{0}}\left(f\left(u_{\varepsilon}\right)+\varepsilon \delta f\left(u_{\varepsilon}\right)-f(u)\right) \partial_{x} \psi d x d t \\
& \quad=-\frac{1}{\varepsilon} \int_{0}^{T}[f(u)]_{\varphi} \int_{\varphi}^{\varphi_{\varepsilon}} \partial_{x} \psi d t d t+\mathcal{O}(\varepsilon) \\
& \quad=-\int_{0}^{T}[f(u)]_{\varphi} \partial_{x} \psi(\varphi, t) \frac{\varphi_{\varepsilon}-\varphi}{\varepsilon} d t d t+\mathcal{O}(\varepsilon) .
\end{aligned}
$$

Substituting (9.26) and (9.27) into (9.25) we obtain (9.20). 


\section{REFERENCES}

1. C. Bardos and O. Pironneau, A formalism for the differentiation of conservation laws, $C . R$. Acad. Sci. Paris, Ser. I, 335 (2002) 839-845. MR.1947710 (2004c:35265)

2. C. Bardos and O. Pironneau, Derivatives and control in presence of shocks, Computational Fluid Dynamics Journal 11(4) (2003) 383-392.

3. C. Bardos and O. Pironneau, Data assimilation for conservation laws, Methods and Applications of Analysis, 12 (2) (2005), 103-134. MR2257523 (2007k:35313)

4. F. Bouchut and F. James, One-dimensional transport equations with discontinuous coefficients, Nonlinear Analysis, Theory and Applications 32(7) (1998) 891-933. MR 1618393 (2000a:35243)

5. F. Bouchut and F. James, Differentiability with respect to inital data for a scalar conservation law, Proceedings of the 7th Int. Conf. on Hyperbolic Problems, Zurich 1998, International Series Num. Math. 129 (Birkhäuser, 1999) 113-118. MR1715739 (2000g:35137)

6. F. Bouchut, F. James and S. Mancini, Uniqueness and weak stability for multi-dimensional transport equations with one-sided Lipschitz coefficient. Ann. Sc. Norm. Super. Pisa Cl. Sci. 4(5) (2005) 1-25. MR2165401 (2006j:35017)

7. F. Bouchut and B. Perthame, Kruzkov's estimates for scalar conservation laws revisited, Transactions of the American Mathematical Society, 350 (7) (1998) 2847-2870. MR 1475677 (98m:65156)

8. Y. Brenier and S. Osher, The discrete one-sided Lipschitz condition for convex scalar conservation laws, SIAM Journal on Numerical Analysis 25(1) (1988) 8-23. MR.923922 (89a:65134)

9. A. Bressan and A. Marson, A variational calculus for discontinuous solutions of systems of conservation laws, Commun. in Partial Differential Equations 20(9-10) (1995) 1491-1552. MR.1349222 (96g:35120)

10. A. Bressan and A. Marson, A maximum principle for optimally controlled systems of conservation laws, Rend. Sem. Mat. Univ. Padova 94 (1995) 79-94. MR.1370904 (97c:49025)

11. C. Castro, F. Palacios and E. Zuazua, An alternating descent method for the optimal control of the inviscid Burgers equation in presence of discontinuities, Mathematical Models and Methods in Applied Science, 18 (3) (2008), 369-416. MR2397976 (2009c:35392)

12. C. Castro, F. Palacios y E. Zuazua, Optimal control and vanishing viscosity for the Burgers equation, in proceedings of Integral Methods in Science and Engineering, Vol. 2, Computational Methods, C. Constanda et al. eds., Birkhäuser Boston, 2010, 65-90.

13. G. Dal Maso, Ph. Le Floch and F. Murat, Definition and weak stability of nonconservative products, J. Math. Pures Appl. 74 (1995) 458-483. MR.1365258 (97b:46052)

14. M. Escobedo, J.L. Vázquez and E. Zuazua, Asymptotic behaviour and source-type solutions for a diffusion-convection equation, Arch. Rat. Mech. Anal. 124(1) (1993) 43-65. MR1233647 $(94 \mathrm{j}: 35077)$

15. S. Garreau, Ph. Guillaume and M. Masmoudi, The topological asymptotic for PDE systems: the elasticity case. SIAM J. Control Optim. 39(6) (2001) 1756-177. MR1825864 (2002m:49062)

16. M.B. Giles and N.A. Pierce, Analytic adjoint solutions for the quasi one-dimensional Euler equations, J. Fluid Mechanics 426 (2001) 327-345. MR.1819479 (2002a:76037)

17. R. Glowinski, Numerical Methods for Fluids (Part 3) Handbook of Numerical analysis, vol. IX (Ph. Ciarlet and J. L. Lions eds., Elsevier, 2003). MR2009814 (2004j:65001)

18. R. Glowinski, J.-L. Lions and R. Trémolières, Analyse Numérique des Inegalités Variationnelles, Vol.1: Théorie Général: Premiéres Applications, Dunod, Paris, 1976. MR0655454 $(58: 31697 \mathrm{a})$

19. E. Godlewski and P.A. Raviart, The linearized stability of solutions of nonlinear hyperbolic systems of conservation laws. A general numerical approach, Mathematics and Computer in Simulations 50 (1999) 77-95. MR.1717658 (2000i:35119)

20. E. Godlewski and P.A. Raviart, Hyperbolic systems of conservation laws, Mathématiques and Applications, (Ellipses, Paris, 1991). MR 1304494 (95i:65146)

21. E. Godlewski, M. Olazabal and P.A. Raviart, On the linearization of hyperbolic systems of conservation laws. Application to stability, Equations différentielles et Applications, articles dédiés à Jacques-Louis Lions (Gauthier-Villars, Paris, 1998) 549-570. MR1648240 (99h:35125) 
22. E. Godlewski and P.A. Raviart, Numerical approximation of hyperbolic systems of conservation laws (Springer-Verlag, 1996). MR,1410987(98d:65109)

23. L. Gosse and F. James, Numerical approximations of one-dimensional linear conservation equations with discontinuous coefficients, Mathematics of Computation 69 (2000) 987-1015. MR 1670896 (2000j:65077)

24. C. Hirsch, Numerical computation of internal and external flows, Vol. 1 and 2 (John Wiley and Sons, 1988).

25. F. James and M. Postel, Numerical gradient methods for flux identification in a system of conservation laws, J. Eng. Math. 60 (2008) 293-317. MR.2396486 (2009b:65211)

26. F. James and M. Sepúlveda, Convergence results for the flux identification in a scalar conservation law. SIAM J. Control Optim. 37(3) (1999) 869-891. MR1680830 (2000b:65171)

27. S. N. Kruzkov, First order quasilinear equations with several space variables, Math. USSR. Sb., 10 (1970) 217-243.

28. R. LeVeque, Finite Volume Methods for Hyperbolic Problems (Cambridge Univesity Press, 2002). MR1925043 (2003h:65001)

29. B. Lucier, A moving mesh numerical method for hyperbolic conservation laws, Math. of Comp. 46(173) (1986), 59-69. MR815831 (87m:65141)

30. A. Majda, The stability of multidimensional shock fronts (AMS, 1983). MR683422 (84e:35100)

31. G. Métivier, Stability of multidimensional shocks, course notes at http://www.math.ubordeaux.fr/ ${ }^{\sim}$ metivier/cours.html (2003).

32. B. Mohammadi and O. Pironneau, Shape optimization in fluid mechanics, Annual Rev. Fluids Mechanics 36 (2004) 255-279. MR2062314 (2005a:76138)

33. S. Nadarajah and A. Jameson, A Comparison of the Continuous and Discrete Adjoint Approach to Automatic Aerodynamic Optimization, AIAA Paper 2000-0667, 38th Aerospace Sciences Meeting and Exhibit, January 2000, Reno, NV.

34. O. Oleinik, Discontinuous solutions of nonlinear differential equations, Uspekhi Mat. Nauk. 12 (1957) 3-73 (In Russian), Amer. Math. Soc. Trans. 26 95-172 (In English). MR0094541 $(20: 1055)$

35. S. Ubrich, Adjoint-based derivative computations for the optimal control of discontinuous solutions of hyperbolic conservation laws, Systems and Control Letters 48 (2003) 313-328. MR2020647 (2005g:49042)

36. K. Yosida, Functional Analysis, (Springer, 1995). MR.1336382 (96a:46001)

Departamento de Matemática e Informática, etsi Caminos, Canales y Puertos, UniVersidad PolitéCnica de Madrid, 28040 Madrid, Spain.

E-mail address: carlos.castro@upm.es

Basque Center for Applied Mathematics (BCAM), Bizkaia Technology Park, Building 500, E-48160 Derio, Basque Country, Spain - And - Ikerbasque, Basque Foundation for Science, E-48011 Bilbao, Basque Country, Spain

E-mail address: zuazua@bcamath.org 